\title{
Molecular Thermodynamics for Salt-Induced Protein Precipitation
}

\author{
D. E. Kuehner, H. W. Blanch and J. M. Prausnitz \\ Department of Chemical Engineering \\ University of California \\ and \\ Chemical Sciences Division \\ Lawrence Berkeley National Laboratory \\ University of California \\ Berkeley, CA 94720, U.S.A.
}

May 1999

This work was supported by the Director, Office of Energy Research, Office of Basic Energy Sciences, Chemical Sciences Division of the U.S. Department of Energy under Contract Number DE-AC03-76SF00098. 


\section{ABSTRACT}

A molecular-thermodynamic model is developed for salt-induced protein precipitation; the model considers an aqueous solution of globular protein molecules as a system of interacting hard spheres in a continuum pseudo-solvent (water and salt ions). The protein molecules interact in a manner described by a set of spherically-symmetric two-body potentials of mean force. These include screened Coulombic repulsion, dispersion (van der Waals) attraction, and ion-excluded-volume osmotic attraction. Specific chemical interactions between proteins (e.g., hydrophobic interactions and self-association) are modeled with a short-range, orientation-dependent attractive square-well potential. An analytical equation of state is derived using the Random Phase Approximation with the hard-sphere fluid as the reference system and a perturbation based on the protein-protein overall potential of mean force, while the short-range specific chemical interaction is incorporated through the SAFT perturbation theory of associating fluids. This equation of state, similar in form to the well-known van der Waals equation of state, provides an expression for the chemical potential of the protein and determines liquid-liquid phase equilibria. Critical and spinodal criteria for salt-induced protein precipitation are derived, and the dependence of critical points and protein solubility on model parameters is examined. Salting-out phase-equilibrium data are described for two globular proteins, henegg-white lysozyme and bovine $\alpha$-chymotrypsin, in solutions of ammonium sulfate at $25^{\circ} \mathrm{C}$. For both proteins, the SAFT parameters $M, \varepsilon_{s p}$, and $V$ are correlated from the lysozyme precipitation data of Coen, et al. (1995). Calculated Cohn-Edsall slopes relating the protein solubility to the solution ionic strength are greater than those measured in precipitation experiments with lysozyme and chymotrypsin, indicating that ionic-strengthdependent parameters are required for quantitative application of this model to these proteins. 


\section{DISCLAIMER}

This report was prepared as an account of work sponsored by an agency of the United States Government. Neither the United States Government nor any agency thereof, nor any of their employees, make any warranty, express or implied, or assumes any legal liability or responsibility for the accuracy, completeness, or usefulness of any information, apparatus, product, or process disclosed, or represents that its use would not infringe privately owned rights. Reference herein to any specific commercial product, process, or service by trade name, trademark, manufacturer, or otherwise does not necessarily constitute or imply its endorsement, recommendation, or favoring by the United States Government or any agency thereof. The views and opinions of authors expressed herein do not necessarily state or reflect those of the United States Government or any agency thereof. 


\section{DISCLAIMER}

Portions of this document may be illegible in electronic image products. Images are produced from the best available original document. 


\section{INTRODUCTION}

Precipitation has long been used as an early step in the process of purifying proteins from complex solutions (Green, 1931; Green, 1932). This technique has been applied to the recovery of proteins such as insulin, diagnostic and industrial enzymes, human growth hormone, and interferon (McGregor, 1983). Phase separation in aqueous protein solutions is achieved through addition of precipitating agents, such as inorganic salts at high concentrations, nonionic polymers, polyelectrolytes, and organic solvents (Foster et al., 1973; Haire et al., 1984; Shih et al., 1992; Rothstein, 1994). Salting-out of proteins provides one of the simplest precipitation techniques and is used extensively in the laboratory and in the biotechnology and pharmaceutical industries.

In most previous studies, salt-induced precipitation of proteins and other colloids has been understood as phase separation resulting in a solid precipitated phase in equilibrium with a liquid phase containing protein at its saturation concentration (Rosenbaum et al., 1996; Tavares and Sandler, 1997). Traditionally, quantitative characterization has been expressed through the protein solubility, defined as the protein concentration in the equilibrium liquid phase. For example, the classical Cohn and Edsall approach (Edsall, 1943) correlates protein solubility, $S$, with solution ionic strength, $I$, in a simple exponential manner: $\log S=\beta-\lambda I$, where $\beta$ and $\lambda$ are empirically-determined parameters specific to a given protein and salt. Recent experimental results (Shih et al., 1992; Coen, 1995) suggest that salt-induced protein precipitation may also be appropriately described as a phase separation resulting in two fluid phases: a supernatant fluid phase lean in protein, in equilibrium with a precipitate phase rich in protein and containing appreciable amounts of water and salt. According to this view, the degree of precipitation is characterized by the distribution coefficient, $K_{e}$, defined as the ratio of the protein concentration in the precipitate phase to that in the supernatant phase. Various experimental equilibrium salting-out studies have shown that protein partitioning can depend strongly on protein size, initial concentration, and surface chemistry; on electrolyte identity and ionic strength; and on solution pH (Arakawa and Timasheff, 1982; Arakawa and Timasheff, 1984). Specific interactions (e.g., dimerization or higher oligomerization, or hydrophobic interactions) may also play a major role in governing protein-protein interactions in precipitating solutions.

To establish a rational basis for designing a protein-precipitation process, it is useful to develop a model to provide a theoretical framework for interpretation and correlation of 
protein-precipitation data. Melander and Horvath showed that the functional form of the Cohn-Edsall equation may be interpreted on the basis of solvophobic (Melander and Horvath, 1977). Recent theoretical studies have been directed at developing more fundamental models to account, on a molecular level, for diverse interactions between the various components in the protein solution. For example, Mahadevan and Hall presented a model, based on Barker-Henderson perturbation theory, for protein precipitation by a nonionic polymer (Mahadevan and Hall, 1990; Mahadevan and Hall, 1992). Vlachy, Blanch and Prausnitz describe a model for polymer-induced liquid-liquid phase separation in solutions of colloids and globular proteins, based on the random-phase approximation (Vlachy et al., 1993). However, these recent theoretical studies are concerned with aqueous solutions where the electrolyte concentration is less than 0.1 molar. Experimental studies clearly show that protein precipitation by salts typically requires electrolyte concentration in the range 1-10 molar.

This work presents a molecular-thermodynamic model for protein precipitation by inorganic salts. Particular attention is given to highly concentrated salt solutions. The procedure employed here represents the ternary solution (protein, electrolyte, and water) as a one-component system containing globular protein molecules immersed in a continuous pseudo-solvent. The pseudo-solvent is an aqueous salt solution. The effect of pseudosolvent chemistry (i.e., $\mathrm{pH}$, salt type and ionic strength) on protein-protein interactions is taken into account through the strong influence that it exerts on the following proteinprotein interactions: Coulombic (charge-charge) repulsion, dispersion (van der Waals) attraction, ion-excluded-volume (or osmotic) attraction, and interactions between exposed hydrophobic groups on the surfaces of two or more protein molecules. Despite its simplicity, the one-component representation has been successful in explaining some experimental properties of colloidal dispersions (Grimson, 1983) and globular-protein solutions (Vlachy et al., 1993; Chiew et al., 1995; Kuehner et al., 1996; Tavares and Sandler, 1997; Curtis et al., 1998). A powerful advantage of this representation is that final results are given by analytical equations based on statistical-mechanical theories.

Two-body potentials of mean force are discussed in Section 2. Section 3 presents a derivation of the molecular-thermodynamic equation-of-state model for protein solutions, based on the random-phase approximation (RPA) and the statistical associating fluid theory (SAFT). Results of model calculations and comparison with experimental precipitation data are presented in Section 4. 


\section{PROTEIN-PROTEIN POTENTIAL OF MEAN FORCE}

In the one-component model, aqueous solutions of globular proteins are represented by a monodisperse assembly of spherical non-interpenetrating spheres of diameter $\sigma_{p}$ which interact through effective solvent-dependent potentials of mean force (PMFs). The overall potential of mean force, $W(r)$, between protein molecules is given by the sum of five potentials of mean force:

$$
W(r)=W_{h s}(r)+W_{q q}(r)+W_{d i s p}(r)+W_{o s m}(r)+W_{x p}(r, \omega)
$$

where $r$ is the center-to-center distance between proteins. Here, $W_{h s}(r)$ is the hard-sphere potential, $W_{q q}(r)$ is the repulsive screened electric-double-layer potential of mean force, $W_{\text {disp }}(r)$ is the attractive dispersion potential of mean force, and $W_{o s m}(r)$ is the attractive potential due to ion-excluded-volume effects. These four terms are spherically symmetric potentials of mean force which depend on the center-to-center distance between proteins, on the properties of the protein molecules, and on the solvent chemistry (e.g., $\mathrm{pH}$ and ionic strength). $W_{s p}(r, \omega)$ represents attractive specific interactions between exposed groups on the surfaces of the proteins; these may include hydrophobic interactions and short-range attractive electrostatic interactions. This potential depends not only on $r$ but also on relative molecular orientation, indicated by $\omega$.

\subsection{Hard-Sphere and Coulombic Potentials}

The hard-sphere and repulsive screened Coulombic potentials of mean force between proteins, normalized with respect to the thermal energy $k_{B} T$, are represented by:

$$
\begin{array}{rlrl}
W_{h s}(r) & =\infty & r & \leq \sigma_{p} \\
& =0 & r & >\sigma_{p} \\
\frac{W_{q q}(r)}{k_{B} T}=\frac{\left(z_{p} e\right)^{2}}{4 \pi \varepsilon_{0} \varepsilon_{r} k_{B} T} \frac{\exp \left[-\kappa\left(r-\sigma_{p}\right)\right]}{r \cdot\left(1+\kappa \sigma_{p} / 2\right)^{2}} & & r>\sigma_{p}
\end{array}
$$


where $k_{B}$ denotes Boltzmann's constant, and $T$ is the absolute temperature; $z_{p} e$ is the charge on the protein, where $z_{p}$ is the protein valence and $e$ is the elementary unit charge; $4 \pi \varepsilon_{0}$ represents the dielectric permittivity of vacuum in units of $\mathrm{C} / \mathrm{Vm}$ and $\varepsilon_{r}$ is the ionicstrength-dependent dielectric constant of the salt-water continuum pseudo-solvent; $\kappa$ is the Debye screening parameter, given by $\kappa^{2}=\left(2 e^{2} N_{A} D /\left(\varepsilon_{o} \varepsilon_{r} k_{B} T\right) ; N_{A}\right.$ is Avogadro's number; and ionic strength $I=0.5 \sum c_{m} z_{m}^{2}$, where $c_{m}$ and $z_{m}$ are the molar concentration and valence of ion $m$, respectively.

The value of $W_{q q}(r)$ depends on the ionic strength of the solution, as shown in Figure 1, where $W_{q q}(r)$ is plotted as a function of the center-to-center distance between protein molecules, $r$, normalized by the protein diameter, $\sigma_{p}$. In Figure $1, \sigma_{p}=50 \AA$ and $z_{p}=10$. As ionic strength increases, the electric double layer surrounding the protein molecule compresses and the effective charge-charge interaction between proteins is screened. Also, the protein valence, $z_{p}$, depends on protonation states of the various constituent amino acids which have hydrogen-ion equilibria; hence the protein will have an effective net charge which depends on the solution $\mathrm{pH}$. Each protein has an isoelectric point, pI, where the net charge is zero; hence, at the $\mathrm{pl}, W_{q q}(r)=0$. The net protein charge is a model parameter obtained from independent experimental data.

The Coulombic potential of mean force accounts for long-range repulsive charge-charge interactions between protein molecules present in dilute concentrations. The derivation of this potential assumes that the charge of the protein is evenly distributed over the surface of the molecule. In reality, protein surface charges, both positive and negative, are discrete and are located at sites possessing hydrogen-ion equilibria. In the dilute-protein limit, where the average separation between protein molecules is large (up to several protein diameters), the approximation of evenly-distributed surface charge is acceptable. However, for concentrated-protein solutions (where precipitation or crystallization may occur), the protein molecules are much more closely packed in solution, and short-range attractive electrostatic interactions between oppositely charged moieties on the protein surface become more important, and this assumption is no longer valid. These specific interactions are accounted for in $W_{s p}(r, \omega)$, as discussed below. 
Furthermore, $W_{q q}(r)$ is derived within the Debye-Hiickel framework using a linearized form of the Poisson-Boltzmann equation for the electrostatic potential around the spherical charged protein molecule; this assumption is valid only in solutions where the ionic strength is $0.1 \mathrm{M}$ or less. Application of $W_{q q}(r)$ to solutions with higher ionic strengths is an extrapolation. However, for ionic strengths greater than approximately $0.5 \mathrm{M}$, this potential is screened so strongly that it has only a negligible contribution to the total potential of mean force. Higher-order electrostatic interactions, i.e., between charges and permanent dipoles, are not considered here, since they are generally much weaker in magnitude than $W_{q q}(r)$ and are strongly screened by salt.

\subsection{Dispersion Potential}

Spontaneous electronic fluctuations within polyatomic molecules or surfaces give rise to induced dipoles within the body, which interact with the induced dipoles in a neighboring body. These van-der-Waals-type forces cause protein molecules to experience a net attractive potential of mean force, also electrostatic in nature. For large bodies such as protein molecules, the magnitude of this attractive force is significant. Following the geometric derivation of Derjaguin, the unretarded attractive dispersion interaction $W_{\text {dlsp }}(r)$ is given by the following expression (Hamaker, 1937):

$$
W_{d i s p}(r)=-\frac{H}{12}\left\{\frac{\sigma_{p}^{2}}{r^{2}-\sigma_{p}^{2}}+\frac{\sigma_{p}^{2}}{r^{2}}+2 \ln \left(1-\frac{\sigma_{p}^{2}}{r^{2}}\right)\right\} \quad r>\sigma_{p}
$$

where $H$ is the Hamaker constant of the protein. This form of the dispersion potential has no explicit dependence on ionic strength or $\mathrm{pH}$. The intrinsic frequency of the electronic fluctuations which give rise to the dispersion force is much greater than the time constant for rearrangement of ions in the double layer and in the solution between the protein molecules. Therefore, dielectric screening of the dispersion attraction is assumed to be negligible (Israelachvili and Adams, 1978), and any effect of ionic strength is contained within the effective Hamaker constant, which is an adjustable model parameter. Furthermore, since $H$ is primarily a function of the electron density of the protein molecules (Israelachvili, 1992), it is expected that most proteins have similar values of $H$ since most have similar electron densities (Nir, 1976). As discussed below, the effective Hamaker constant of henegg-white lysozyme can be obtained from light-scattering measurements. Combination of 
$W_{q q}(r)$ and $W_{d i s p}(r)$ form the basis of the Dergajuin-Landau-Verwey-Overbeek (DLVO) theory of colloidal stability (Verwey and Overbeek, 1948).

\subsection{Ion-Excluded-Volume Potential}

In concentrated electrolyte solutions, ions occupy a significant fraction of the total solution volume. When two protein molecules approach contact, ions are excluded from a region between the protein particles; the geometry of this mechanism is shown in Figure 2. The resulting imbalance in the local osmotic pressure exerted by the ions on the proteins gives rise to a short-range attractive force. The area of the plane of ion exclusion can be calculated in terms of the diameter of the protein, $\sigma_{p}$, the average diameter of a salt ion, $\sigma_{i o n}$, and the center-to-center separation, $r$. Multiplying this area by the osmotic pressure of the corresponding protein-free salt solution, $\Pi_{o s m}$, yields the force. When integrated with respect to $r$, the ion-excluded volume potential, $W_{o s m}(r)$, is given by (Asakura and Oosawa, 1954; Asakura and Oosawa, 1958):

$$
\begin{array}{rlrl}
\frac{W_{o s m}(r)}{k_{B} T} & =-\frac{4 \pi}{3} \sigma_{p s}^{3} \cdot\left(\Pi_{o s m}\right) \cdot\left[1-\frac{3 r}{4 \sigma_{p s}}+\frac{r^{3}}{16 \sigma_{p s}^{3}}\right] & & r<\left(\sigma_{p}+\sigma_{p}\right) \\
& =0 & r \geq\left(\sigma_{p}+\sigma_{p}\right)
\end{array}
$$

where $\sigma_{p s}=\left(\sigma_{p}+\sigma_{i o n}\right) / 2$ and the composition-weighted average ion diameter $\sigma_{i o n}=$ $\left(v_{c a t} \sigma_{c a t}+v_{a n} \sigma_{a n}\right) / v$, where $v_{c a t}$ and $v_{a n}$ are the stoichiometric coefficients of the cation and anion, respectively, and $v=v_{c a t}+v_{a n}$. The osmotic pressure of the protein-free salt solution is obtained from the literature in terms of the osmotic coefficient, $\Phi_{o s m}$, which is a measure of the non-ideality of the salt solution, by $\Pi_{o s m}=\Phi_{o s m} \Pi_{i d}=\Phi_{o s m} \cdot\left(\rho_{s} k_{B} T\right)$, where the total ion number density, $\rho_{s}$, is given in terms of the molar salt concentration, $c_{s}$, by $\rho_{s}=c_{s} N_{A} v . W_{o s m}(r)$ is a short-range attractive potential of mean force which becomes significant at high ionic strength, as shown in Figure 3. In Figure 3, protein diameter $\sigma_{p}=$ $30 \AA$. The salt is ammonium sulfate: hydrated ionic diameters are $\sigma_{c a t}=6.62 \AA$ and $\sigma_{a n}=$ 
$7.58 \AA$ (Horvath, 1985), and values of the osmotic coefficient, $\Phi_{o s m}$, for ammonium sulfate solutions were taken from the literature (Clegg et al., 1996) (see Appendix B). In the derivation of equation (4), salt ions are considered uncharged hard spheres; electrostatic effects are assumed to be taken into account in $\Phi_{o s m}$ and $W_{q q}(r)$.

Figure 4 shows the spherically-symmetric potential of mean force, $W_{s y m}(r)$, composed of $W_{q q}(r)+W_{\text {disp }}(r)+W_{o s m}(r)$, as a function of $r / \sigma_{p}$ for several values of ammonium-sulfate ionic strength. In Figure 4, $\sigma_{p}=30 \AA, z_{p}=15$ and $H / k_{B} T=5$, and the salt is ammonium sulfate. At low ionic strength a repulsive barrier exists due to $W_{q q}(r)$. As ionic strength increases, $W_{q q}(r)$ diminishes due to dielectric screening, and the magnitude of the ionexcluded-volume potential $W_{o s m}(r)$ increases. For ionic strengths greater than $1.0 \mathrm{M}$, $W_{s, m}(r)$ is uniformly attractive with a steep attractive well near surface contact; at extremely high ionic strengths, this represents a strong driving force for protein aggregation and precipitation.

The effect of solution $\mathrm{pH}$ on $W_{\text {smm }}(r)$ is solely through the $\mathrm{pH}$ dependence of the protein valence, $z_{p}$, in the screened Coulombic repulsion potential of mean force. Hence, any $\mathrm{pH}$ dependence of $W_{\mathrm{ssm}}(r)$ diminishes as ionic strength increases. In dilute protein solutions at low ionic strength, $W_{\text {smm }}(r)$ describes well the $\mathrm{pH}$ dependence of the osmotic second virial coefficient (Curtis et al., 1998) and of the average apparent diffusion coefficient of proteins in aqueous solutions (Kuehner et al., 1997). However, protein-precipitation data in concentrated salt solutions often show a significant dependence on solution $\mathrm{pH}$ (Shih et al., 1992; Coen, 1995) beyond that captured in $W_{s m}(r)$. As discussed below, the specific interaction potential, $W_{s p}(r, \omega)$, contains parameters which are $\mathrm{pH}$ dependent and are obtained from experimental data.

\subsection{Specific Interactions}

Specific interactions between proteins are known to occur in solutions where crystallization or precipitation phase transitions occur. These additional forces include hydrophobic interactions and short-ranged electrostatic interactions between surface groups, which depend on the relative orientations of the two protein molecules. The hydrophobic interaction between between exposed non-polar amino-acid residues on the surfaces of the protein molecules is, in general, attractive, short-ranged, and orientation-dependent. 
Hydrophobic "bonds" are formed when hydrophobic groups on separate protein molecules come into contact and may cause association or aggregation of proteins. Similarly, attractive charge-charge couplings between oppositely-charged groups on the surfaces of two protein molecules, as well as salt bridges in solutions with divalent ions, can form in concentrated protein solutions. Although short-ranged, these can affect protein solubility.

In this work, specific interactions are represented by a potential model used for associating fluids (Jackson et al., 1988). The shapes of these specific-interaction sites are idealized as "circular patches" or "spherical caps" located on the surface of the protein molecules. As indicated in Figure 5, interaction potential $W_{s p}(r, \omega)$ between site $A$ on the surface of protein 1 and site $B$ on the surface of protein 2 is defined as an orientation-dependent square-well potential:

$$
\begin{aligned}
\frac{W_{s p}(r, \omega)}{k_{B} T} & =-\frac{\varepsilon_{s p}}{k_{B} T} & & \text { if } r<r_{c} \text { and } \theta_{1} \leq \theta_{l, c} \text { and } \theta_{2} \leq \theta_{2, c} \\
& =0 & & \text { otherwise }
\end{aligned}
$$

Hence, two "patches" on two different particles are considered to form a "bond" if the centers of the two particles are within a critical distance, $r_{c}$, and if the two patches are within the constraints of relative orientation, defined by the critical angles $\theta_{I, c}$ and $\theta_{2, c^{*}}$. In general, these quantities are not known. Fortunately, the thermodynamic properties of systems with this type of interaction can be described in terms of quantities that are known or can be estimated from physicochemical data for proteins, as described below.

\section{LIQUID-STATE THEORY AND EQUATION OF STATE}

Having established and defined pertinent potentials of mean force, it is necessary to construct a molecular-thermodynamic model which relates these potentials to macroscopic thermodynamic properties. In this work, that model is based on perturbation theory. The center-to-center spherically symmetrical Coulombic, dispersion and ion-excluded-volume interactions are incorporated into the model in the context of the random-phase approximation (RPA), described in Section 3.1. The contribution of the orientationdependent specific interaction potential is included through a first-order perturbation theory of associating fluids, the statistical associating fluid theory (SAFT), described in Section 
3.2. The RPA-SAFT equation of state, which combines both these contributions, is presented in Section 3.3.

\subsection{Random-Phase Approximation}

The Random-Phase Approximation (RPA) has been used previously to model the structure factor of colloid solutions (Grimson, 1983) and to describe liquid-liquid phase separation of proteins due to addition of polymers (Mahadevan and Hall, 1990; Mahadevan and Hall, 1992; Vlachy et al., 1993). In the RPA, an assembly of hard spheres is used as the reference system, while the spherically symmetric interactions are treated as perturbations. Thermodynamic properties of the system are obtained from liquid-state integral-equation theory. The basis of this framework is the Ornstein-Zernike (OZ) equation describing two-body interactions between particles in a monodisperse, isotropic fluid (Ornstein and Zernike, 1914):

$$
h\left(r_{12}\right)=c\left(r_{12}\right)+\rho \int c\left(r_{13}\right) h\left(r_{23}\right) d r_{23}
$$

In this formal representation, the interaction between molecule 1 and molecule 2 in the fluid, characterized by the total correlation function $h\left(r_{12}\right)$, is composed of two contributions: the direct interaction, given by the direct correlation function $c\left(r_{12}\right)$, and the indirect interactions transmitted from 1 to 2 through interaction with one other molecule (labeled molecule 3 ). Higher order terms, for instance interactions between molecules 1 and 2 transmitted through two other particles (i.e., molecules 3 and 4) are assumed negligible. Therefore, the physical meaning of the $\mathrm{OZ}$ equation is that the total interaction for a two-body interaction is composed of the direct correlation, plus the density-weighted sum of 1-2 interactions transmitted through all other particles 3 . Here, $\rho$ is the number density (number of molecules per unit volume) of the molecular fluid. Through equation (2.6) the interactions between all molecules in the fluid are coupled. In a monodisperse isotropic fluid, $r_{12}, r_{13}$ and $r_{23}$ are replaced simply by $r$, the center-to-center distance between molecules. The direct correlation function $h(r)$ is related to the potential of mean force between molecules through the following definition (McQuarrie, 1976):

$$
h(r)+1=g(r)=\exp \left[-W_{s y m}(r) / k_{B} T\right]
$$


where $g(r)$ is the pair-correlation function, or radial distribution function, which relates the spatially-varying concentration of molecules in the fluid, $\rho(r)$, to the average concentration $\rho$, by the following: $\rho(r)=\rho g(r)$.

The connection of the $\mathrm{OZ}$ equation to thermodynamic properties of solutions comes from performing a Fourier transformation on equation (6), resulting in:

$$
1-\rho \hat{C}(k)=\frac{1}{1+\rho \hat{H}(k)}
$$

where $\hat{C}(k)$ and $\hat{H}(k)$ represent the Fourier analogs of $c(r)$ and $h(r)$, respectively. By Fourier-transforming in the limit $k=0$ (which corresponds to taking a volume integral), the thermodynamic pressure and chemical potential may be obtained from the isothermal compressibility relation for a one-component fluid:

$$
1-\rho \hat{C}(0)=\frac{1}{k_{B} T}\left(\frac{\partial P}{\partial \rho}\right)_{T}=\frac{\rho}{k_{B} T}\left(\frac{\partial \mu}{\partial \rho}\right)_{T}
$$

The random-phase approxirnation consists of defining the direct correlation function, $c(r)$, as:

$$
c(r)=c_{h s}(r)-W_{s y m}(r) / k_{B} T
$$

where $c_{h s}(r)$ is the direct correlation function for the hard-sphere reference system. This is most valid in the limit of small $W_{\text {ssm }}(r)$ and low molecular concentration, $\rho$. Fourier transformation of equation (10) in the $k=0$ limit and integration of equation (9) with respect to $\rho$ yields the following expressions for the pressure and residual chemical potential of protein in solution:

$$
\begin{aligned}
& \frac{P_{R P A}}{\rho k_{B} T}=\frac{P_{h s}}{\rho k_{B} T}+\frac{\rho U}{2 k_{B} T} \\
& \frac{\mu_{r e s, R P A}}{k_{B} T}=\frac{\mu_{h s}}{k_{B} T}+\frac{\rho U}{k_{B} T}
\end{aligned}
$$


where $P_{h s}$, and $\mu_{h s}$ are the pressure and chemical potential, respectively, of the hard-sphere reference system. The contributions to the pressure and chemical potential from the spherically-symmetric interactions are given by the terms in equations (11) and (12) containing the RPA perturbation energy, $U$, defined as the volume integral of the spherically-symmetric potential of mean force:

$$
U=4 \pi \int_{\sigma_{p}}^{\infty} W_{s y m}(r) \cdot r^{2} d r
$$

Because $U$ is an energy per unit density, it is independent of protein concentration; $U$ depends only on potentials of mean force between protein molecules. The hard-sphere reference terms are given by the standard Carnahan-Starling forms (Carnahan and Starling, 1970):

$$
\begin{aligned}
& \frac{P_{h s}}{\rho k_{B} T}=\frac{1+\eta+\eta^{2}-\eta^{3}}{(1-\eta)^{3}} \\
& \frac{\mu_{h s}}{\rho k_{B} T}=\frac{\eta\left(8-9 \eta+3 \eta^{2}\right)}{(1-\eta)^{3}}
\end{aligned}
$$

where the protein packing fraction $\eta=(\pi / 6) \rho \sigma_{p}^{3}$. This theory is applied to proteins in aqueous salt solutions within the context of the McMillan-Mayer solution theory (Hill, 1960), in which the potential of mean force between particles immersed in a solvent is analogous to the pair potential for non-ideal gases interacting through a vacuum.

\subsection{Statistical Associating Fluid Theory}

The contribution of the orientation-dependent specific interactions to the residual Helmholtz energy and pressure of the system are evaluated using the first-order perturbation theory of associating fluids formulated by Wertheim (Wertheim, 1986a; Wertheim, 1986b), extended to mixtures by Jackson and co-workers (Jackson et al., 1988). At a given protein number density, $\rho$, the Statistical Associating Fluid Theory (SAFT) gives the residual Helmholtz free energy of the associating system relative to that of the non-associating reference 
system. The reference system is an assembly of non-aggregating protein molecules that interact through the spherically-symmetric potential of mean force, $W_{\text {smm }}(r)$.

For a protein having $M$ equivalent exposed specific-interaction sites interacting via the potential given in equation (5), the first-order perturbation theory yields the following contribution to the residual Helmholtz free energy due to hydrophobic association (Jackson $e t$ al., 1988):

$$
a_{\text {res,assod }} d k_{B} T=M\{\ln X+(1-X) / 2]
$$

Here, $X$ represents the fraction of specific-interaction sites which do not participate in a bonding interaction:

$$
X=\frac{-1+\sqrt{1+4 M \rho \Delta}}{2 M \rho \Delta}
$$

where

$$
\Delta=g\left(\sigma_{p}\right) \cdot\left[\exp \left(\varepsilon_{s p} / k_{B} T\right)-1\right] \cdot V
$$

and

$$
V=\frac{3\left[1-\cos \left(\theta_{1, c}\right)\right]\left[1-\cos \left(\theta_{2, c}\right)\right]}{2 \pi} \cdot \frac{\left(r_{c}-\sigma_{p}\right)}{\sigma_{p}}
$$

where $g\left(\sigma_{p}\right)$ is the radial distribution function of the non-aggregating system, and $\varepsilon_{s p}$ represents the characteristic energy of the specific interaction. Parameter $V$ corresponds to the volume of interaction of a site, expressed as a fraction of the total protein volume. The function $g\left(\sigma_{p}\right)$ for non-aggregating proteins (which interact through the sphericallysymmetric potential of mean force) is estimated using the EXP approximation (Konior and Jedrzejek, 1985):

$$
g\left(\sigma_{p}\right)=g_{h s}\left(\sigma_{p}\right) \cdot \exp \left[-W_{s y m}\left(\sigma_{p}\right) / k_{B} T\right]
$$


where $g_{h s}\left(\sigma_{p}\right)$ is given by the contact value of the Carnahan-Starling expression for the hard-sphere radial distribution function:

$$
g_{k s}\left(\sigma_{p}\right)=\frac{1-\eta / 2}{(1-\eta)^{3}}
$$

The first-order perturbation theory of association assumes that the interactions between specific-interaction sites on different molecules are independent and that no ring structures are formed. The standard thermodynamic relation $P=-(\partial A / \partial V)_{T}$ yields the associatingfluid contribution to the total thermodynamic pressure:

$$
\frac{P_{a s s o c}}{\rho k_{B} T}=M \eta\left(\frac{1}{X}-\frac{1}{2}\right) \cdot\left(\frac{\partial X}{\partial \eta}\right)_{T}
$$

The associating-fluid contribution to the residual chemical potential of protein is obtained from equations (16) and (22):

$$
\frac{\mu_{\text {res, assoc }}}{k_{B} T}=M\left\{\ln X+\frac{1-X}{2}+\eta\left(\frac{\partial X}{\partial \eta}\right)_{T}\left(\frac{1}{X}-\frac{1}{2}\right)\right\}
$$

\subsection{Equation of State for Associating Protein Molecules}

Combining equations (11) - (23) using the thermodynamic relation $\mu_{r e s} / k_{B} T=a_{r e s} / k_{B} T+$ $P / \rho k_{B} T$ - 1 yields the RPA-SAFT equation of state and expressions for the residual Helmholtz free energy and excess chemical potential of aqueous protein solutions:

$$
\begin{aligned}
& \frac{P}{\rho k_{B} T}=\frac{1+\eta+\eta^{2}-\eta^{3}}{(1-\eta)^{3}}+\frac{\rho U}{2 k_{B} T}+M \eta\left(\frac{1}{X}-\frac{1}{2}\right) \cdot\left(\frac{\partial X}{\partial \eta}\right)_{T} \\
& \frac{a_{r e s}}{k_{B} T}=\frac{4 \eta-3 \eta^{2}}{(1-\eta)^{2}}+\frac{\rho U}{2 k_{B} T}+M\left[\ln X+\frac{1-X}{2}\right]
\end{aligned}
$$




$$
\frac{\mu-\mu^{*}}{k_{B} T}=\frac{\eta\left(8-9 \eta+3 \eta^{2}\right)}{(1-\eta)^{3}}+\frac{\rho U}{k_{B} T}+M\left\{\ln X+\frac{1-X}{2}+\eta\left(\frac{1}{X}-\frac{1}{2}\right) \cdot\left(\frac{\partial X}{\partial \eta}\right)_{T}\right\}+\ln \rho
$$

Here, the ideal-gas chemical potential $\mu^{i} / k_{B} T=\mu^{*} / k_{B} T+\ln \rho$, where $\mu^{*}$ is a function only of temperature: $\mu^{*} / k_{B} T=\ln \left(\Lambda^{3}\right)$; the deBroglie wavelength $\Lambda=h / \sqrt{2 \pi m k_{B} T}$, where $h$ is Planck's constant and $m$ is the mass of the protein molecule.

The number of specific-interaction sites, $M$, the interaction energy, $\varepsilon_{s p}$, and the interaction volume, $V$, can be estimated from relevant physicochemical data for the protein of interest or correlated from a reasonably small set of precipitation data, as described below.

\section{PHASE EQUILIBRIUM IN PROTEIN SOLUTIONS}

\subsection{Critical Points and Spinodal and Coexistence Curves}

The spinodal criterion for a one-component solution is (Prausnitz et al., 1986):

$$
-\left(\frac{\partial^{2} A}{\partial V^{2}}\right)_{T}=\left(\frac{\partial P}{\partial \rho}\right)_{T}=0
$$

where $A$ is the Helmholtz free energy of protein and $V$ is system volume. At the critical point, in addition to equation (27), the following criterion also applies:

$$
-\left(\frac{\partial^{3} A}{\partial V^{3}}\right)_{T}=\left(\frac{\partial^{2} P}{\partial \rho^{2}}\right)_{T}=0
$$

The partial differentials of the pressure with respect to density for the RPA-SAFT equation of state (24) are derived in Appendix A. At equilibrium, protein concentrations in the supernatant and dense-fluid phases are calculated from equations (24) and (26) based on the classical equilibrium conditions: 


$$
\begin{aligned}
& \mu_{s}=\mu_{d} \\
& P_{s}=P_{d}
\end{aligned}
$$

Here, subscripts $s$ and $d$ denote the equilibrium supernatant and dense protein phases, respectively. In equation (29a), $\mu^{*}$ cancels out.

Figure 6 shows thermodynamic pressure calculated from the RPA-SAFT equation of state, normalized by $\sigma_{p}^{3} / k_{B} T$, as a function of protein packing fraction, $\eta$, for several values of ionic strength. For this case, the parameters are: $\sigma_{p}=50 \AA, H / k_{B} T=10, M=6, \varepsilon_{s p} / k_{B} T=$ $2, V=0.02$, and the salt is ammonium sulfate. For ionic strength below the critical point, a unique P- $\eta$ relationship exists. However, for higher ionic strengths there are two values of $\boldsymbol{\eta}$ having the same pressure, corresponding to two coexisting equilibrium liquid phases. The additional constraint of equality of chemical potentials, equation (29a), defines the actual values of the equilibrium phase compositions.

Figure 7 shows typical spinodal and coexistence curves for the RPA-SAFT equation of state. The parameters in this figure are the same as for Figure 6 . The critical ionic strength for liquid-liquid phase equilibrium, $I_{c}$, is $2.22 \mathrm{M}$ in this case, and the critical protein volume fraction, $\eta_{c}$, is 0.171 . For solutions with ionic strength greater than $I_{c}$ and protein concentrations which correspond to values of $\eta$ which lie between the two branches of the spinodal curve (where $\partial P / \partial \eta<0$ and the solution is thermodynamically unstable), the solution separates into two liquid phases having protein concentrations $\eta_{s}$ and $\eta_{d}$, in the supernatant and dense phase, respectively, obtained from the coexistence curve at the specified solution ionic strength.

Figures $8(a)$ and $8(b)$ show the dependence of the critical ionic strength, $I_{c}$, on the SAFT parameters $M, \varepsilon_{s p}$ and $V$, and on the protein diameter, $\sigma_{p}$, respectively, for a hypothetical protein in ammonium sulfate solution. (In Figure $8(a), V$ is represented as $V \%=V \cdot 100$, for convenience in graphing.) For these calculations, the base set of parameters is the same as in Figures 6 and 7; the parameter indicated in the figure legend was varied, while all the other parameters were held constant at the values given above. Figure 8(a) shows that as 
the value of any of the SAFT parameters increases, attractive specific interactions increase, and hence the critical ammonium sulfate ionic strength required to induce precipitation decreases. The strength of the specific interaction, $\varepsilon_{s p}$, and the number of interaction sites, $M$, have a stronger effect on $I_{c}$ than does the interaction volume, $V$. The protein diameter, $\sigma_{p}$, has the strongest effect on $I_{c}$ : for a protein with diameter of $30 \AA, I_{c}=9 \mathrm{M}$, while for a $60 \AA$ protein, only $1 \mathrm{M}$ ammonium-sulfate ionic strength is required to cause phase separation. This is due entirely to ion-excluded-volume forces. Consider Figure 2: as the protein diameter increases, the plane from which ions are excluded also increases in area when the two protein molecules approach surface contact. Hence, there is a larger area over which the osmotic pressure imbalance acts, increasing the attractive force at any given ionic strength and resulting in a lower $I_{c}$ required to induce precipitation. This phenomenon -- that larger proteins precipitate at lower ionic strength than smaller proteins -- has been previously observed in experimental salting-out protein-precipitation studies (A.lbertsson, 1986; Shih et al, 1992; Coen, 1995).

For precipitating systems, two quantities of primary interest in comparing model predictions to experimental phase-equilibrium data are: the supernatant protein concentration, often equated with the protein apparent solubility, $S$, expressed here in terms of the supernatant-phase protein packing fraction, $\eta_{s}$ (recall, $\eta_{s}=\pi / 6 \rho_{s} \sigma_{p}^{3}$, where $\rho_{s}$ is the protein number concentration in the supernatant phase); and the equilibrium partition coefficient, $K_{e}$, defined as $K_{e}=\eta_{d} / \eta_{s}$, where $\eta_{d}$ is the equilibrium protein packing fraction in the dense (precipitated) phase. Figures 9 through 12 examine the effect of varying the SAFT parameters and the protein diameter on calculated phase-equilibrium compositions. Again, the base set of parameters is the same as in Figures 6,7 and 8; the parameter indicated in the figure legend was varied, while all the other parameters were held constant at the values given above. In Figures 9 and 10, an increase in ionic strength results in a decrease in $\eta_{s}$, indicating greater partitioning into the dense phase. Furthermore, as any of the SAFT parameters increases at a given ionic strength, the magnitude of the attractive specific interactions increases and the equilibrium supernatant protein packing fraction decreases. As shown in Figure 10(b), protein diameter, $\sigma_{p}$, has the most significant effect on protein solubility, and interaction volume, $V$, has the weakest influence. 
Figures 11 and 12 show that the equilibrium protein partition coefficient, $K_{e}$, increases exponentially with increasing ionic strength. As shown in Figures 9 and 10, increasing the values of the SAFT parameters increases the amount of attraction between protein molecules and results in higher protein partition coefficients. Similarly, larger proteins show much greater partitioning than smaller proteins at any given ionic strength above the critical value. Also, Figures 11 and 12 show that the RPA-SAFT model has the power to describe equilibrium protein partition coefficients with values from 1 (near the critical point) up to values on the order of $10^{4}$, which corresponds to essentially complete protein precipitation, using reasonable values of model parameters at experimentally relevant salt concentrations.

\subsection{Hen-Egg-White Lysozyme Precipitation}

Figure 13 shows experimental precipitation data (Coen, 1995) for hen-egg-white lysozyme (points) in ammonium-sulfate solutions, for $\mathrm{pH}$ between 5 and 8 and ionic strength between 5 and 9 molal, along with values of $K_{e}$ calculated from the RPA-SAFT equation of state for the same solutions (dotted lines). While the experimental equilibrium partition coefficient increases with ionic strength, no dependence on $\mathrm{pH}$ is observed at any ionic strength shown in Figure 13. The potential-of-mean-force model incorporates $\mathrm{pH}$ dependence only through the screened Coulombic repulsion potential, $W_{q q}(r)$. For ionic strengths above 1 molal, the contribution of long-range macromolecular Coulombic repulsion to the total spherically-symmetric potential of mean force, $W_{\mathrm{sm}}(r)$, is negligible due to dielectric screening; therefore, the RPA contribution to the thermodynamic properties of the system is independent of $\mathrm{pH}$.

However, equilibrium partitioning at high ionic strength does depend on the solution $\mathrm{pH}$ for most proteins. This includes lysozyme: at $\mathrm{pH}<5$, lysozyme $K_{e}$ increases significantly with decreasing pH at all ionic strengths [data not shown; see (Coen, 1995)]. Chymotrypsin partition coefficient also increases with decreasing $\mathrm{pH}$, as shown in Figure 16(a). There are several possible physicochemical reasons for this observation. Partial denaturation in acidic conditions may make the protein more prone to precipitate. As the protein denatures, its effective hard-sphere diameter $\sigma_{p}$ increases. As shown in Figure 12(b), for a given ionic strength, larger proteins precipitate more readily than smaller proteins. Also, with partial denaturation comes the possibility of greater solvent exposure of hydrophobic amino-acid groups which are normally sequestered in the interior of the protein, when in its 
native conformation. Increased hydrophobic interactions can enhance protein aggregation and precipitation. Furthermore, at low pH, significant anion binding may occur (Carr, 1953; Praybycien and Bailey, 1989), altering the surface chemistry and attenuating short-range electrostatic interactions. In high-ionic-strength solutions, there may be little free water of hydration available to the protein molecules. Desolvation of the protein can lead to conformational shifts and changes in surface chemistry, which may provide an ionicstrength dependence to protein partitioning beyond what is accounted for in the sphericallysymmetric potential of mean force. Whether this change favors increased or decreased partitioning depends on the specific protein and salt under consideration ${ }^{1}$. In the context of the RPA-SAFT description of salting-out phase equilibrium, all these protein- and saltspecific effects are accounted for by correlating $\mathrm{pH}$ - and/or ionic-strength-dependent values of the SAFT parameters from a set of experimental precipitation data.

In applying the RPA-SAFT model to precipitation data of the type shown in Figure 13, physical insight is required in choosing appropriate values of the SAFT parameters $M, \varepsilon_{s p \text {, }}$ and $V$. Here, $M$ is assigned the value of 6 for lysozyme, enabling the formation of large,

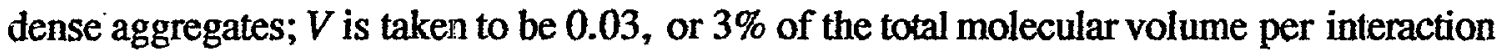
site. The specific-interaction energy, $\varepsilon_{s p}$, is allowed to vary and is regressed from the experimental precipitation data. The potential-of-mean-force parameters for lysozyme are taken from earlier work (Kuehner et al., 1997): the Hamaker constant (which depends on neither $\mathrm{pH}$ nor ionic strength), $H / k_{B} T=8.9$, and the protein net valence, $z_{p}$ is obtained from extrapolation of lysozyme titration curves titration curves (Kuehner et al., 1998).

Figure 14 shows the values of the specific-interaction energy, $\varepsilon_{s p}$, regressed from the average values of the equilibrium protein partition coefficient, $K_{e, a v g}$, shown in Figure 13. $\varepsilon_{s p}$ decreases in a linear fashion with increasing ionic strength. This is an ernpirical regression and reflects the "true" interactions occurring in this system only to the extent that the physics included in the RPA-SAFT model are correct. Within these physical assumptions, Figure 14 implies that as ionic strength increases, ion-excluded-volume forces grow rapidly enough that a decrease in the specific-interaction energy is required to

\footnotetext{
${ }^{1}$ In addition, specific ion effects on protein interactions and solubility have been well studied and analyzed theoretically (Hofmeister, 1888; Carr, 1953; Carr, 1953; Melander and Horvath, 1977; Arakawa and Timasheff, 1982; Arakawa and Timasheff, 1984; Arakawa and Timasheff, 1985; Ries-Kautt and Ducruix, 1989; Ries-Kautt and Ducruix, 1994; Coen, 1995; Curtis et al., 1998) but are not discussed in the context of this work, where ammonium sulfate is the only salt examined.
} 
describe accurately the experimental equilibrium protein partition coefficient in ammoniumsulfate solutions.

Figure 15 examines the ionic-strength dependence of the equilibrium protein partition coefficient of lysozyme in ammonium-sulfate solutions at $\mathrm{pH}$ 8.0. Figure 15(a) shows experimental values of $K_{e}$ in logarithmic scale, along with calculated partition coefficients for two different values of $\varepsilon_{s p}$, where $\varepsilon_{s p}$ is held constant with respect to ionic strength. Both the experimental and the calculated values of $K_{e}$ follow classical Cohn-Edsall behavior (Edsall, 1943), where the protein solubility (expressed here as $K_{e}$ ) exhibits an exponential dependence on the ionic strength. However, the slope of the experimental line is smaller than that of either of the two calculated lines. The values of $K_{e}$ calculated using $\varepsilon_{s p} / k_{B} T=$ 4.0 match the experimental values better at lower ionic strengths but overpredict partitioning as ionic strength approaches 9 molal. Conversely, the values of $K_{e}$ calculated using $\varepsilon_{s p} / k_{B} T=3.8$ match the experimental values well at high ionic strength, but underpredict partitioning at lower ionic strengths. This subtlety is not easily seen in Figure 15(b), where the same values of $K_{e}$ are displayed on a linear scale. However, Figure 15(b) shows the sensitivity of calculated values of $K_{e}$ to $\varepsilon_{s p}$ at high ionic strength more effectively than does Figure 15(a). Figure $15(b)$ also illustrates that when high degrees of precipitation are desired (which would presumably be one of the potential applications of such a model), it is more important to fit model parameters to high-ionic-strength data than to low-ionic-strength data. In order to make calculated values fit experimental values more exactly over the whole range of salt concentration, $\varepsilon_{s p}$ must be allowed to vary with ionic strength.

\subsection{Bovine $\alpha_{-}$-Chymotrypsin Precipitation}

Bovine $\alpha$-chymotrypsin is a serine protease involved in the degradation of other proteins in the digestive process. It is composed of 245 amino acids in three chains joined by disulfide linkages between cysteine residues. As with lysozyme, chymotrypsin's structure has been characterized by $\mathrm{X}$-ray crystallography: in its native conformation, it is a compact globular molecule, slightly prolate, with axes $51 \times 40 \times 40 \AA$, giving an equivalent spherical diameter $\sigma_{p}=43.4 \AA$; it has molecular weight $24,500 \mathrm{Da}$ and isoelectric point 8.3 (Stryer, 1988). 
Figure 16(a) shows the dependence of the equilibrium protein partition coefficient, $K_{e}$, on solution $\mathrm{pH}$ and ionic strength, for precipitation of $\alpha$-chymotrypsin in ammonium-sulfate solutions. For chymotrypsin, precipitation is significantly enhanced as $\mathrm{pH}$ decreases, whereas $K_{e}$ for lysozyme showed no $\mathrm{pH}$ dependence. Figure $16(b)$ shows calculated and experimental values of $K_{\varepsilon}$ in logarithmic format. For the RPA-SAFT calculations with chymotrypsin, $M$ was again taken as 6 . Chymotrypsin is larger than lysozyme (MW = $14,500 \mathrm{Da} ; \sigma_{p}=34.4 \AA$ ). Hence, the volume fraction of each specific-interaction site in chymotrypsin was chosen to be slightly smaller than in lysozyme; here, $V=0.02$, corresponding to $2 \%$ of the protein volume per site. The Hamaker constant of chymotrypsin, $H / k_{B} T=10$, a value similar to that of lysozyme. The Hamaker constant depends primarily on the electron density of the macromolecule and is therefore expected to have similar values for most small globular proteins (Nir, 1976). The net valence of chymotrypsin was obtained from titration experiments (Fergg, 1994). Figure 16(b) shows that, as is the case for lysozyme precipitation in ammonium-sulfate solutions, the RPASAFT model gives calculated Cohn-Edsall slopes for chymotrypsin that are slightly higher than the experimental values. Consequently, values of $\varepsilon_{s p}$ which vary with ionic strength, as well as with $\mathrm{pH}$, must be used to correlate the data of Coen (Coen, 1995) for chymotrypsin precipitation with quantitative accuracy.

Comparison of Figures 15 and 16 shows that lysozyme precipitates at lower ammoniumsulfate ionic strength than chymotrypsin, although lysozyme is the smaller of the two proteins. Normally larger proteins are observed to salt out at lower ionic strength than smaller proteins (Albertsson, 1986; Rothstein, 1994); this observation is also reflected in RPASAFT calculations, as shown in Figure 12(b). Attractive specific interactions (or, at least, interactions not included in $W_{\text {smm }}(r)$, the spherically symmetric potential of mean force) in lysozyme solutions must be responsible for lysozyme's lower solubility in ammonium sulfate. This is highlighted by the difference in the correlated values of the specificinteraction energies: for chymotrypsin, $\varepsilon_{s p} / k_{B} T \sim 1$, while for lysozyme, $\varepsilon_{s p} / k_{B} T \sim 4$. These additional forces may arise from, for example, differences in the extent of hydrophobic interaction, differences in protein-salt interactions, and/or conformational changes in precipitating solutions. 


\section{CONCLUSION}

An approximate statistical-mechanical equation-of-state model has been derived to describe salt-induced protein precipitation. In this model, protein-protein interactions are quantified through potentials of mean force (PMFs) which account for electrostatic repulsion, dispersion attraction, ion-excluded-volume attraction, and hydrophobic interactions. Thermodynamic properties of the system are derived using two perturbation theories: spherically symmetric interactions are described by the random phase approximation (RPA), and orientation-dependent attractive specific interactions (e.g., hydrophobic interactions, short-range electrostatic interactions or self-association) are accounted for using the statistical associating fluid theory (SAFT).

The equation of state has been applied to solutions of hypothetical proteins in aqueous solutions of ammonium sulfate. Calculated phase-equilibrium results show that both the supernatant-phase protein concentration, $\eta_{s}$, and the equilibrium protein partition coefficient, $K_{e}$, exhibit exponential dependence on ionic strength. Cohn and Edsall (Edsall, 1943) were the first to make this observation experimentally and to correlate salting-out solubilities for a variety of proteins using a simple, purely empirical exponential formula; successfully describing this phenomenon is one of the first requirements of a thermodynamic model for protein precipitation. Protein partitioning is most sensitive to protein size, with larger proteins being more prone to precipitate than smaller proteins, in a solution of any given ionic strength. Furthermore, attractive specific interactions may play an important role in the precipitation of proteins at high salt concentrations; the SAFT contribution to precipitation is a strong function of the magnitude of the specific-interaction energy, $\varepsilon_{s p}$. It has been shown in principle that, using the RPA-SAFT equation of state, either ion-excluded-volume or specific-interaction forces, or both, can be used to interpret protein-precipitation data.

The RPA-SAFT equation of state has been applied to real protein-precipitation data for henegg-white lysozyme and bovine $\alpha$-chymotrypsin in concentrated ammonium-sulfate solutions. Potential-of-mean force parameters for hen-egg-white lysozyme from previous work (Kuehner et al., 1997) are used to calculate the RPA contributions to the thermodynamic pressure and chemical potential of protein in precipitating solutions. Precipitation of both proteins is described well using six specific-interaction sites. Quantitative agreement between experimental and calculated values of the protein equilibrium partition coefficient, 
$K_{e}$, is obtained by using values of the specific-interaction energy, $\varepsilon_{s p}$, which depend on ionic strength for lysozyme and on both ionic strength and $\mathrm{pH}$ for chymotrypsin. In general, the strength of attractive specific interactions was found to be greater for lysozyme than for chymotrypsin at all solution conditions.

In summary, the RPA-SAFT equation of state provides simple analytic expressions for the thermodynamic properties of soluble and precipitating solutions of globular proteins. It includes contributions from a variety of intermolecular interactions which depend on solution $\mathrm{pH}$ and ionic strength, two variables often used to control protein precipitation in practice. With five adjustable parameters which may be correlated from a reasonable number of light-scattering, titration and protein-precipitation experiments, the RPA-SAFT equation of state provides quantitative description of equilibrium protein partitioning in precipitation systems. In the future, it is expected that incorporation of additional significant features -- e.g., specific protein-ion interaction effects, multi-component expressions describing the properties of mixtures of proteins, and application to protein crystallization -will significantly enhance the utility of this type of molecular-thermodynamic model in biochemical engineering applications. 


\section{APPENDIX A: Spinodal and Critical Points in Aqueous Protein Solutions}

For a one-component fluid, the spinodal criterion is:

$$
-\left(\frac{\partial^{2} A}{\partial V^{2}}\right)_{T}=\left(\frac{\partial P}{\partial \rho}\right)_{T}=Z+\eta \cdot\left(\frac{\partial Z}{\partial \eta}\right)_{T}=0
$$

where $A$ is the Helmholtz free energy of protein and $V$ is system volume; the packing fraction of protein $\eta=(\pi / 6) \rho \sigma_{p}^{3}$, where $\rho$ is the protein number density and $\sigma_{p}$ is the protein effective hard-sphere diameter. The compressibility factor, $Z$, may be written as the sum of three contributions:

$$
Z=P l \rho k_{B} T=Z_{h s}+Z_{R P A}+Z_{a s s o c}
$$

where:

$$
\begin{aligned}
& Z_{h s}=\frac{1+\eta+\eta^{2}-\eta^{3}}{(1-\eta)^{3}} \\
& Z_{R P A}=\frac{\rho U}{2 k_{B} T} \\
& Z_{\text {assoc }}=M \eta\left(\frac{1}{X}-\frac{1}{2}\right)\left(\frac{\partial X}{\partial \eta}\right)_{T}
\end{aligned}
$$

where $U$ is the RPA perturbation energy (per unit density), $k_{B}$ is Boltzmann's constant and $T$ is absolute temperature. The association parameters $M$ and $X$ represent the number of equivalent bonding sites on the protein molecule and the fraction of unbound sites, respectively, where $X$ is given by:

$$
X=\frac{-1+\sqrt{1+4 M \rho \Delta}}{2 M \rho \Delta}
$$


and

$$
\Delta=\frac{1-\eta / 2}{(1-\eta)^{3}} \cdot \exp \left(-\frac{W\left(\sigma_{p}\right)}{k_{B} T}\right) \cdot\left[\exp \left(\frac{\varepsilon_{s p}}{k_{B} T}\right)-1\right] \cdot V
$$

Differentiation with respect to $\eta$ yields:

$$
\begin{aligned}
& \frac{\partial Z_{h s}}{\partial \eta}=\frac{2\left(2+2 \eta-\eta^{2}\right)}{(1-\eta)^{4}} \\
& \frac{\partial Z_{R P A}}{\partial \eta}=\frac{U}{2 k_{B} T} \cdot \frac{6}{\pi \sigma_{p}^{3}} \quad \text { noting that } \rho=6 \eta /\left(\pi \sigma_{p}^{3}\right) \\
& \frac{\partial Z_{a s s o c}}{\partial \eta}=M\left[\left(\frac{1}{X}-\frac{1}{2}\right)\left(\frac{\partial X}{\partial \eta}+\eta \frac{\partial^{2} X}{\partial \eta^{2}}\right)-\frac{\eta}{X^{2}}\left(\frac{\partial X}{\partial \eta}\right)^{2}\right]
\end{aligned}
$$

where the constant-temperature notation has been omitted for convenience on the density derivatives. The derivatives of $X$ with respect to $\eta$ are given by:

$$
\begin{aligned}
& \frac{\partial X}{\partial \eta}=J \cdot\left(\frac{1}{\sqrt{1+4 M \rho \Delta}}-X\right) \\
& \frac{\partial^{2} X}{\partial \eta^{2}}=\left(\frac{1}{J} \frac{\partial J}{\partial \eta}-J\right) \frac{\partial X}{\partial \eta}-J^{2} \frac{2 M \rho \Delta}{(\sqrt{1+4 M \rho \Delta})^{3}}
\end{aligned}
$$

where

$$
J=\frac{3}{1-\eta}-\frac{1}{2-\eta}+\frac{1}{\eta}
$$




$$
\frac{\partial J}{\partial \eta}=\frac{3}{(1-\eta)^{2}}-\frac{1}{(2-\eta)^{2}}-\frac{1}{\eta^{2}}
$$

Thus, at the spinodal point, equation (A.1) becomes:

$$
\begin{aligned}
& \frac{1+4 \eta+4 \eta^{2}-4 \eta^{3}+\eta^{4}}{(1-\eta)^{4}}+\frac{\rho U}{k_{B} T}+ \\
& M \eta\left[\left(\frac{1}{X}-\frac{1}{2}\right)\left(2 \frac{\partial X}{\partial \eta}+\eta \frac{\partial^{2} X}{\partial \eta^{2}}\right)-\frac{\eta}{X^{2}}\left(\frac{\partial X}{\partial \eta}\right)^{2}\right]=0
\end{aligned}
$$

At the critical point the additional constraint

$$
-\left(\frac{\partial^{3} A}{\partial V^{3}}\right)_{T}=\left(\frac{\partial^{2} P}{\partial \rho^{2}}\right)_{T}=0
$$

is imposed. Equation (A.16) is equivalent to:

$$
2 \frac{\partial Z}{\partial \eta}+\eta \frac{\partial^{2} Z}{\partial \eta^{2}}=0
$$

Again, differentiation with respect to $\eta$ yields:

$$
\begin{aligned}
& \frac{\partial^{2} Z_{h s}}{\partial \eta^{2}}=\frac{4\left(5+2 \eta-\eta^{2}\right)}{(1-\eta)^{5}} \\
& \frac{\partial^{2} Z_{R P A}}{\partial \eta^{2}}=0
\end{aligned}
$$




$$
\begin{aligned}
\frac{\partial^{2} Z_{\text {assoc }}}{\partial \eta^{2}}=M\left(\frac{1}{X}-\frac{1}{2}\right) & \left(2 \frac{\partial^{2} X}{\partial \eta^{2}}+\eta \frac{\partial^{3} X}{\partial \eta^{3}}\right) \\
& -\frac{M}{X^{2}} \frac{\partial X}{\partial \eta}\left[2 \frac{\partial X}{\partial \eta}\left(1-\frac{\eta}{X} \frac{\partial X}{\partial \eta}\right)+3 \eta \frac{\partial^{2} X}{\partial \eta^{2}}\right]
\end{aligned}
$$

where

$$
\begin{aligned}
\frac{\partial^{3} X}{\partial \eta^{3}}=\frac{\partial^{2} X}{\partial \eta^{2}}\left(\frac{1}{J} \frac{\partial J}{\partial \eta}-J\right) & +\frac{\partial X}{\partial \eta}\left[\frac{1}{J} \frac{\partial^{2} J}{\partial \eta^{2}}-\frac{\partial J}{\partial \eta}\left(\frac{1}{J^{2}} \frac{\partial J}{\partial \eta}-1\right)\right] \\
& -\frac{2 M \rho \Delta J}{(1+4 M \rho \Delta)^{3 / 2}}\left[2 \frac{\partial J}{\partial \eta}+J^{2}\left(\frac{1-2 M \rho \Delta}{1+4 M \rho \Delta}\right)\right]
\end{aligned}
$$

and

$$
\frac{\partial^{2} J}{\partial \eta^{2}}=\frac{6}{(1-\eta)^{3}}-\frac{2}{(2-\eta)^{3}}+\frac{2}{\eta^{3}}
$$

Combining, the additional critical criterion (A.17) becomes:

$$
\begin{aligned}
\frac{4 \eta\left(2+5 \eta-\eta^{2}\right)}{(1-\eta)^{5}}+\frac{\rho U}{k_{B} T}+ & M\left(\frac{1}{X}-\frac{1}{2}\right) \cdot\left(2 \frac{\partial X}{\partial \eta}+4 \eta \frac{\partial^{2} X}{\partial \eta^{2}}+\eta^{2} \frac{\partial^{3} X}{\partial \eta^{3}}\right) \\
& -\frac{M \eta}{X^{2}} \cdot \frac{\partial X}{\partial \eta} \cdot\left[2 \frac{\partial X}{\partial \eta} \cdot\left(2-\frac{\eta}{X} \frac{\partial X}{\partial \eta}\right)+3 \eta \frac{\partial^{2} X}{\partial \eta^{2}}\right]=0
\end{aligned}
$$

Given a potential of mean force and values for the association-potential parameters, as discussed in the body of this chapter, equations (A.15) and (A.23) are used to calculate spinodal and critical points in aqueous protein solutions. 


\section{APPENDIX B: Osmotic Coefficients for $\left(\mathrm{NH}_{4}\right)_{2} \mathrm{SO}_{4}$ Solutions}

In evaluating the ion-excluded volume potential of mean force, $W_{o s m}(r)$, the osmotic pressure, $\Pi_{o s m}$, of the corresponding protein-free salt solution is required. The ideal and real osmotic pressures of an aqueous electrolyte solution are related by:

$$
\Pi_{o s m}=\Pi_{l d} \Phi_{o s m}
$$

where the ideal osmotic pressure is:

$$
\Pi_{i d}=c_{\delta} N_{A}\left(v_{c a t}+v_{a n}\right) \bullet k_{B} T
$$

where $c_{s}$ is the molar concentration of salt, $N_{A}$ is Avogadro's number, $v_{\text {cat }}$ and $v_{a n}$ are the stoichiometric coefficients of the cation and anion, respectively, $k_{B}$ is Boltzmann's constant, and $T$ is absolute temperature.

Osmotic coefficients for ammonium-sulfate solutions at $T=298 \mathrm{~K}$ are reported in the literature (Clegg et al., 1996) and are shown in Figure B.1. For use in calculating $W_{o s m}(r)$, the data of Clegg and coworkers were fit to a sixth-order polynomial function, yielding:

$$
\begin{aligned}
\Pi_{\text {osm }}= & 0.000142 m^{6}-0.002788 m^{5}+0.021813 m^{4}-0.088118 m^{3} \\
& +0.204384 m^{2}-0.26135 m+0.768580
\end{aligned}
$$

with a high degree of accuracy $\left(R^{2}=0.998\right)$. 
NOTATION

$a$

$c_{i j}(r)$

$c_{i}$

e

$g_{i j}(r)$

$h_{i j}(r)$

H

$h$

I

$K_{e}$

$k_{B}$

$m_{i}$

M

$N_{\mathrm{A}}$

$P$

$r$

$S$

T

$U$

V

$W(r)$

$z_{i}$

Helmholtz energy, J/mol

direct correlation function of $i j$ pair

concentration of species $i, \mathrm{~mol} / \mathrm{L}$

elementary charge, $1.602 \times 10^{-19} \mathrm{C}$

radial distribution function of $\ddot{j} j$ pair

total correlation function of $\ddot{j}$ pair

Hamaker's constant, J

Planck's constant, $6.6252 \times 10^{-34} \mathrm{~J}$-sec

ionic strength of solution, $\mathrm{mol} / \mathrm{L}$

distribution coefficient

Boltzmann's constant, $1.3804 \times 10^{-23} \mathrm{~J} / \mathrm{K}$

molecular mass of species $i$

number of attractive specific-interaction sites per protein molecule

Avogadro's number, $6.023 \times 10^{23} \mathrm{~mol}^{-1}$

thermodynamic pressure, $\mathrm{Pa}$

center-to-center distance, $\AA$

protein solubility, $\mathrm{mol} / \mathrm{L}$

absolute temperature, $\mathrm{K}$

RPA perturbation energy, $\mathrm{J}-\mathrm{L} / \mathrm{mol}$

specific-interaction volume fraction

potential of mean force

valence of species $i$

\section{Greek Symbols}

$\beta$

Cohn-Edsall salting-out intercept

$\varepsilon_{r}$

relative permittivity

$\varepsilon_{0}$

permittivity of vacuum, C/Vm

$\varepsilon_{s p}$

association energy

$\eta$

protein packing fraction

$\theta$

angle 


$\begin{array}{ll}\kappa & \text { Debye length, } \AA^{-1} \\ \lambda & \text { Cohn-Edsall salting out slope } \\ \mu & \text { chemical potential } \\ v_{i} & \text { stoichiometric coefficient of species } i \\ \Pi_{o s m}, \Pi_{i d} & \text { real, ideal osmotic pressure of protein-free salt solution, } \mathrm{Pa} \\ \rho_{i} & \text { number concentration of species } i \\ \sigma_{i} & \text { diameter of species } i, \AA \\ \sigma_{i o n} & \text { composition-weighted average hydrated ion diameter } \\ \Phi_{o s m} & \text { osmotic coefficient of protein-free salt solution }\end{array}$




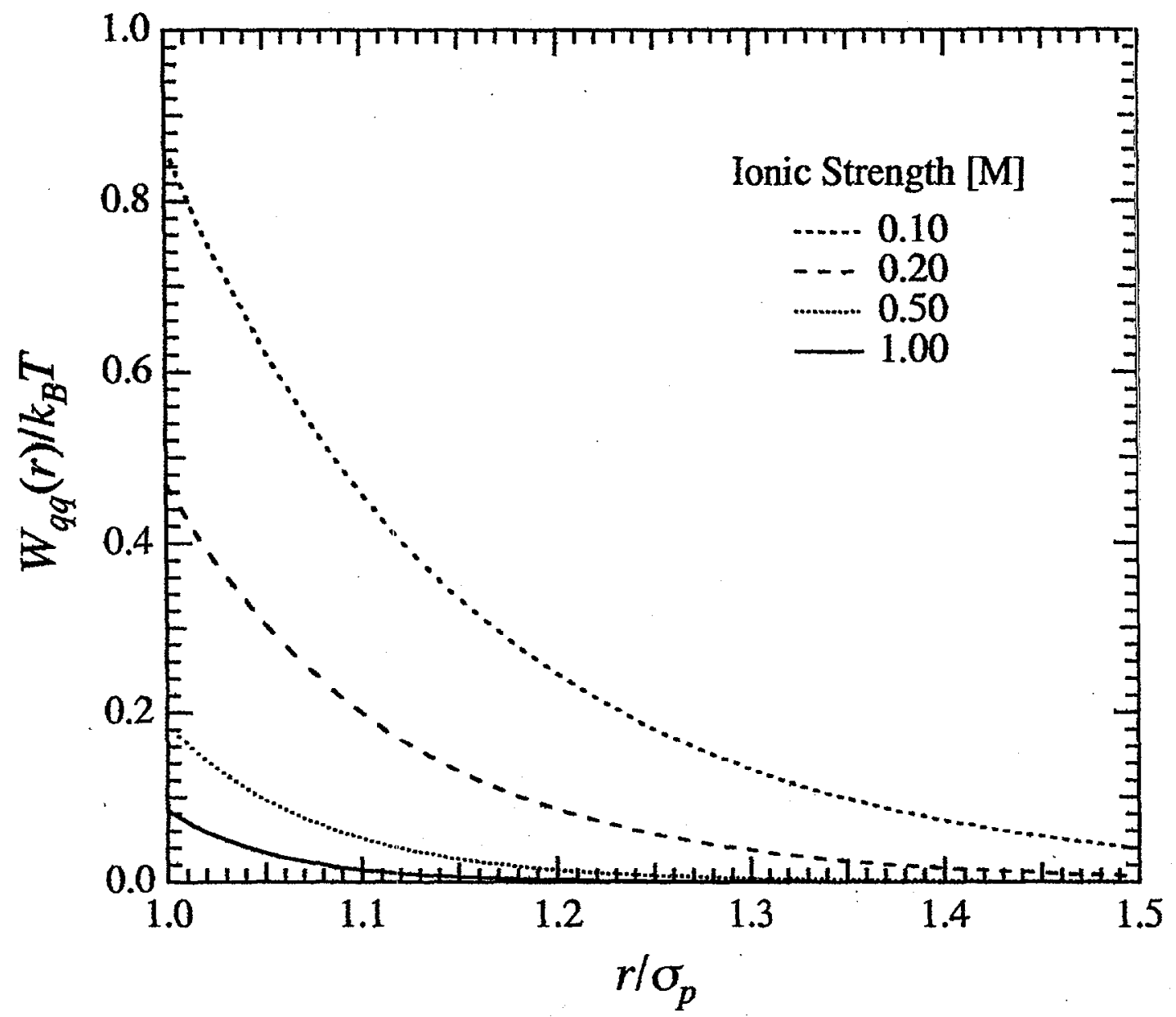

Figure 1: Dependence of the screened Coulombic potential of mean force, $W_{q q}(r)$, on center-to-center separation between protein monomers, $r$, and on solution ionic strength, $I$, in aqueous ammonium sulfate solutions.

Protein parameters: $\quad \sigma_{p}=50 \AA, z_{p}=10$

Salt parameters: $\quad \sigma_{c a t}=6.62 \AA, z_{c a t}=1$

$$
\sigma_{a n}=7.58 \AA, z_{a n}=-2
$$




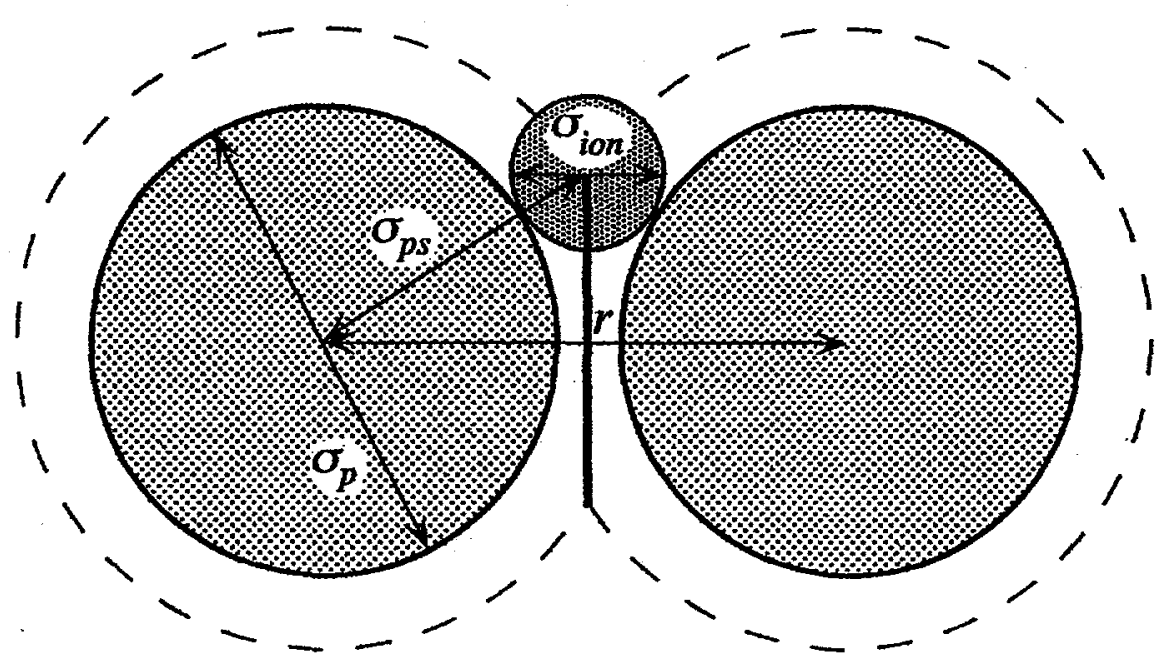

Figure 2: Geometry of the ion-excluded-volume attraction mechanism. 


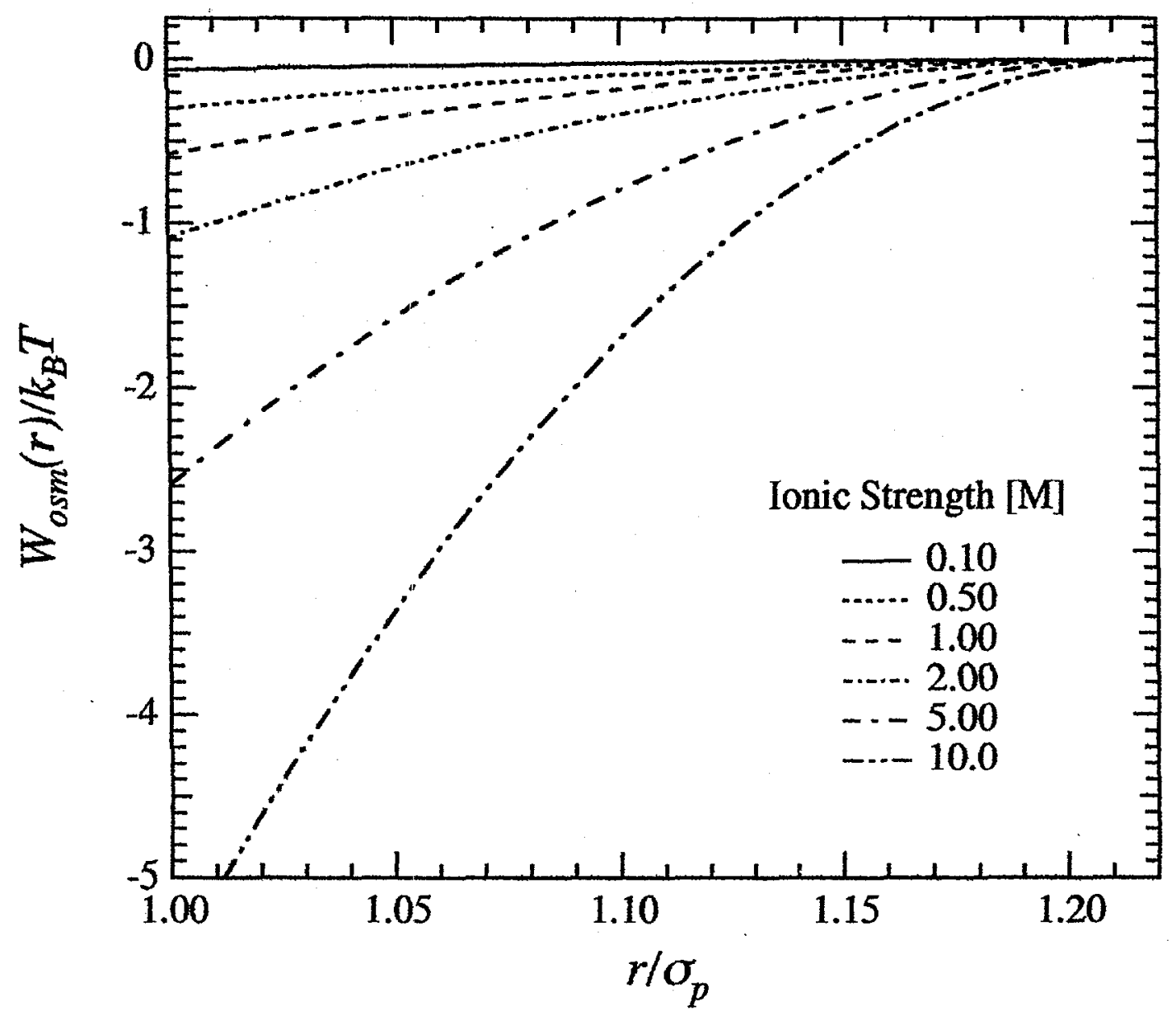

Figure 3: Dependence of the ion-excluded-volume potential of mean force, $W_{o s m}(r)$, on center-to-center separation between protein monomers, $r$, and on solution ionic strength, $I$, in aqueous ammonium sulfate solutions.

Protein parameters: $\quad \sigma_{p}=30 \AA$

Salt parameters:

$$
\begin{aligned}
& \sigma_{p}=30 \AA \\
& \sigma_{c a t}=6.62 \AA, z_{c a t}=1 \\
& \sigma_{a n}=7.58 \AA, z_{a n}=-2
\end{aligned}
$$




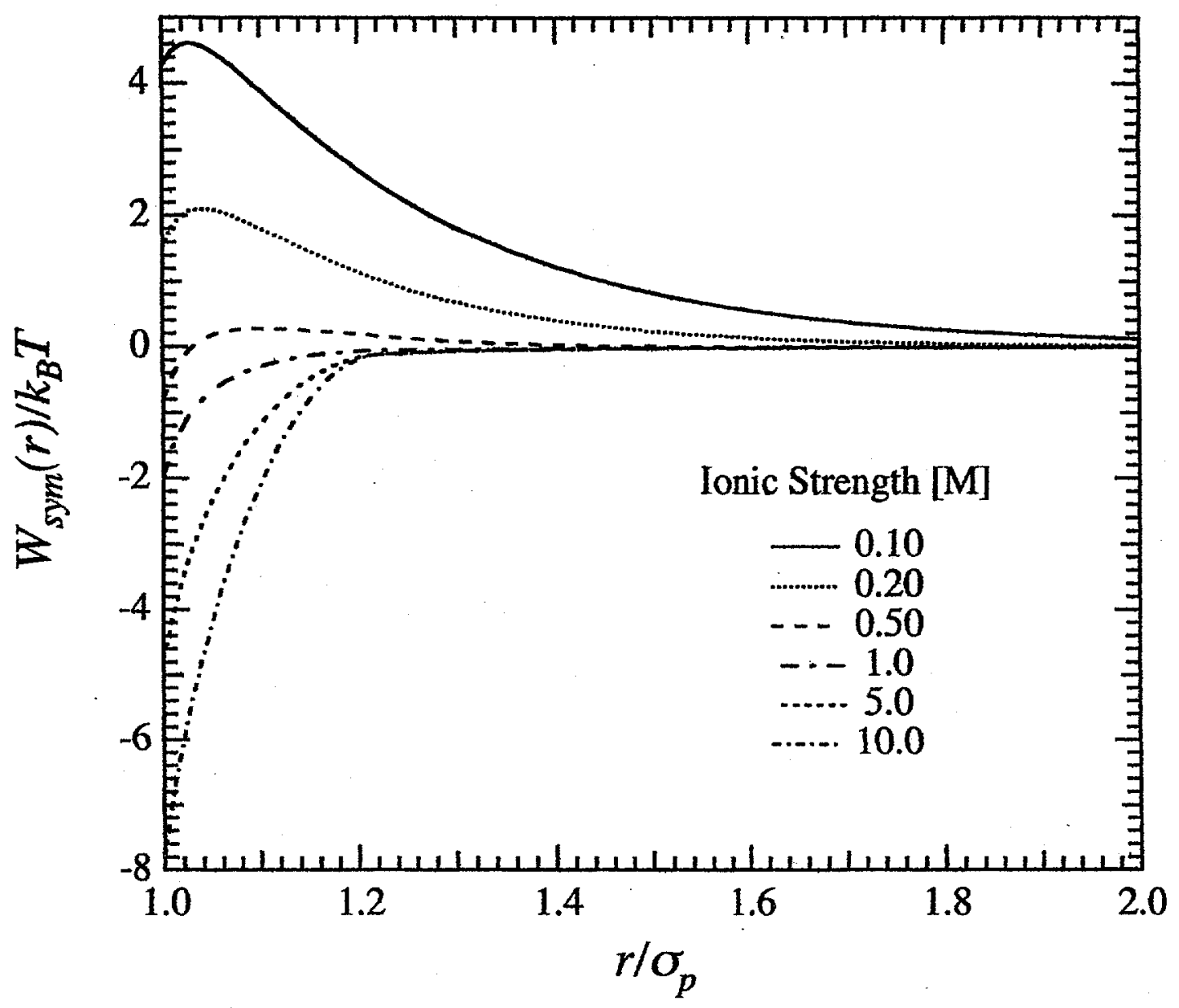

Figure 4: Dependence of the spherically symmetric potential of mean force, $W_{s y m}(r)$, on center-to-center separation between protein monomers, $r$, and on solution ionic strength, $I$, in aqueous ammonium sulfate solutions.

Protein parameters: $\quad \sigma_{p}=30 \AA, z_{p}=15, H / k_{B} T=5$

Salt parameters.

$$
\begin{aligned}
& \sigma_{p}=30 \AA, z_{p}=15, H / k_{B} T=5 \\
& \sigma_{c a t}=6.62 \AA, z_{c a t}=1 \\
& \sigma_{a n}=7.58 \AA, z_{a n}=-2
\end{aligned}
$$




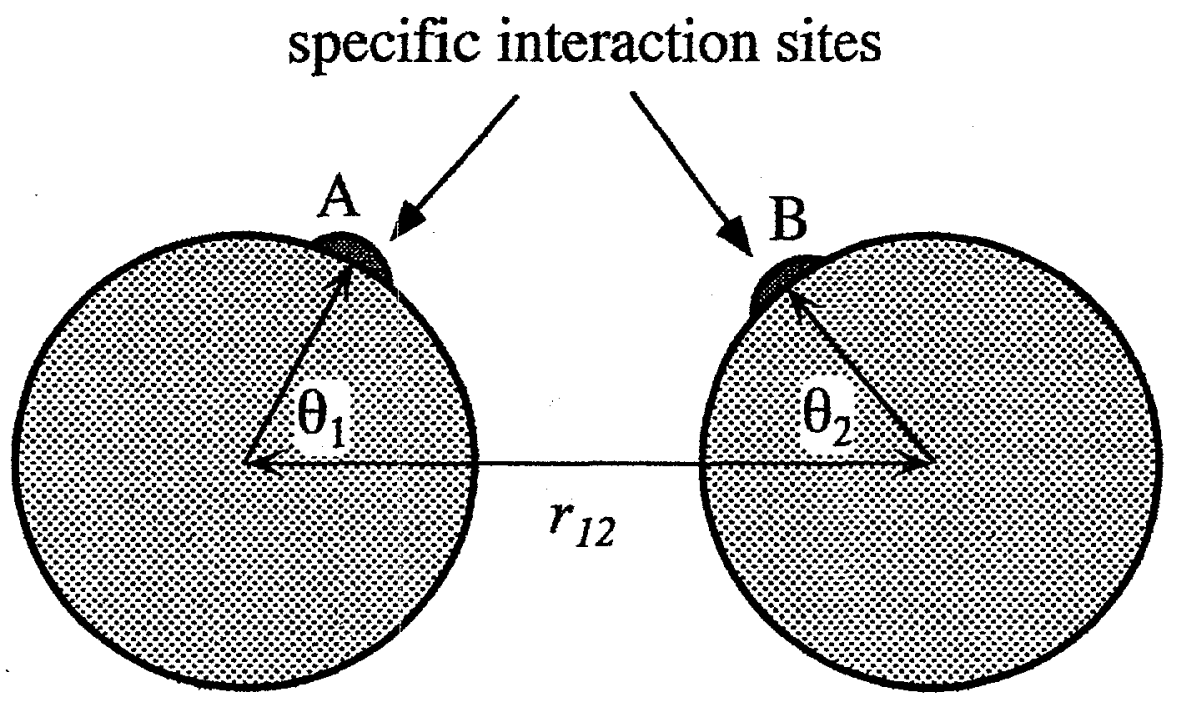

Figure 5: Representation of specific-interaction sites on the protein surface. 


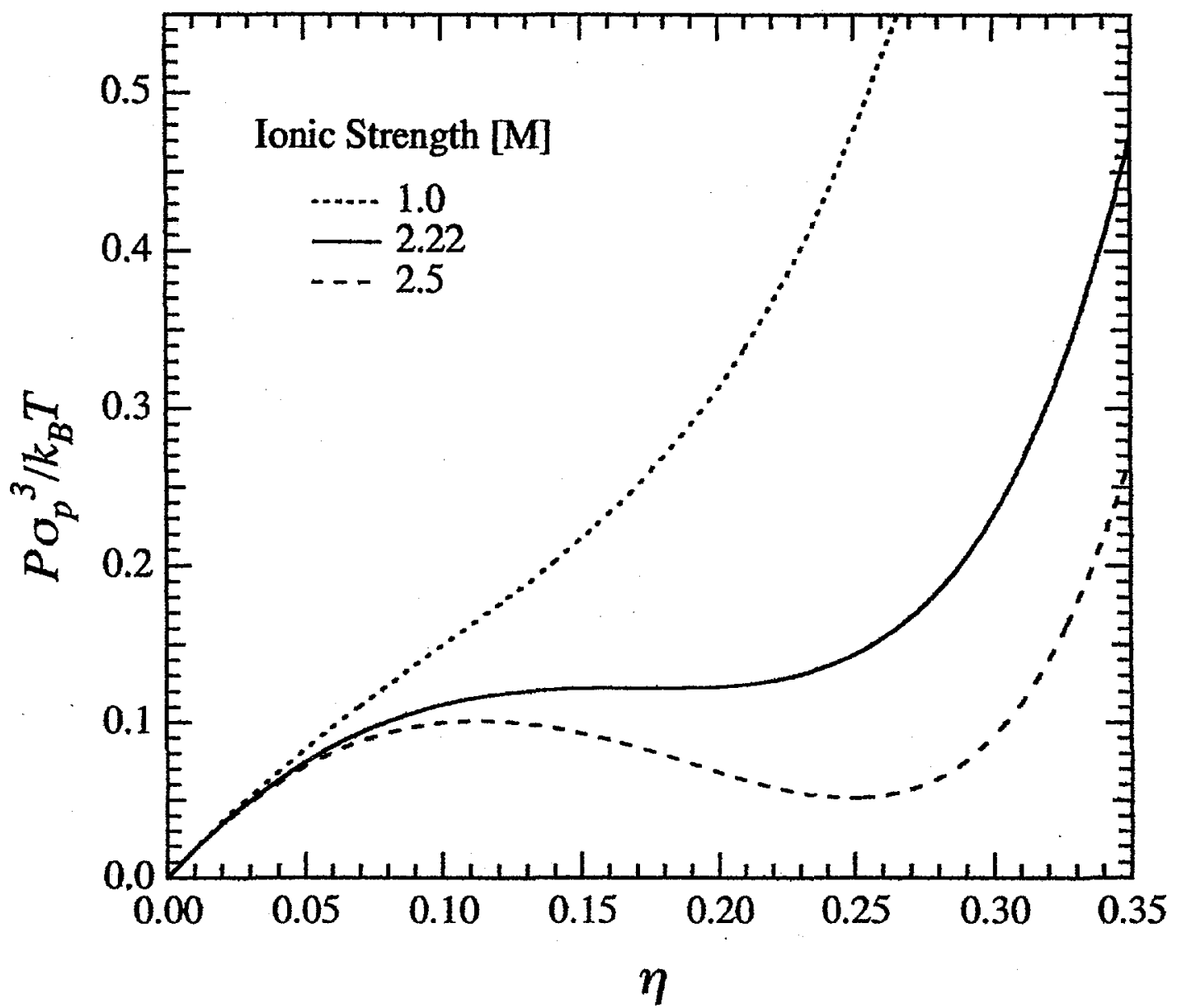

Figure 6: Thermodynamic pressure, normalized by $\sigma_{p}^{3} / k_{B} T$, as a function of protein packing fraction, $\eta$, for three different values of ionic strength. Here, the critical value of ionic strength, $I_{c}$, is $2.22 \mathrm{M}$.

Protein parameters: $\quad \sigma_{p}=50 \AA, H / k_{B} T=10, M=6, \varepsilon_{s p} / k_{B} T=2, V=0.02$

Salt parameters: $\quad \sigma_{c a t}=6.62 \AA, z_{c a t}=1$

$\sigma_{a n}=7.58 \AA, z_{a n}=-2$ 


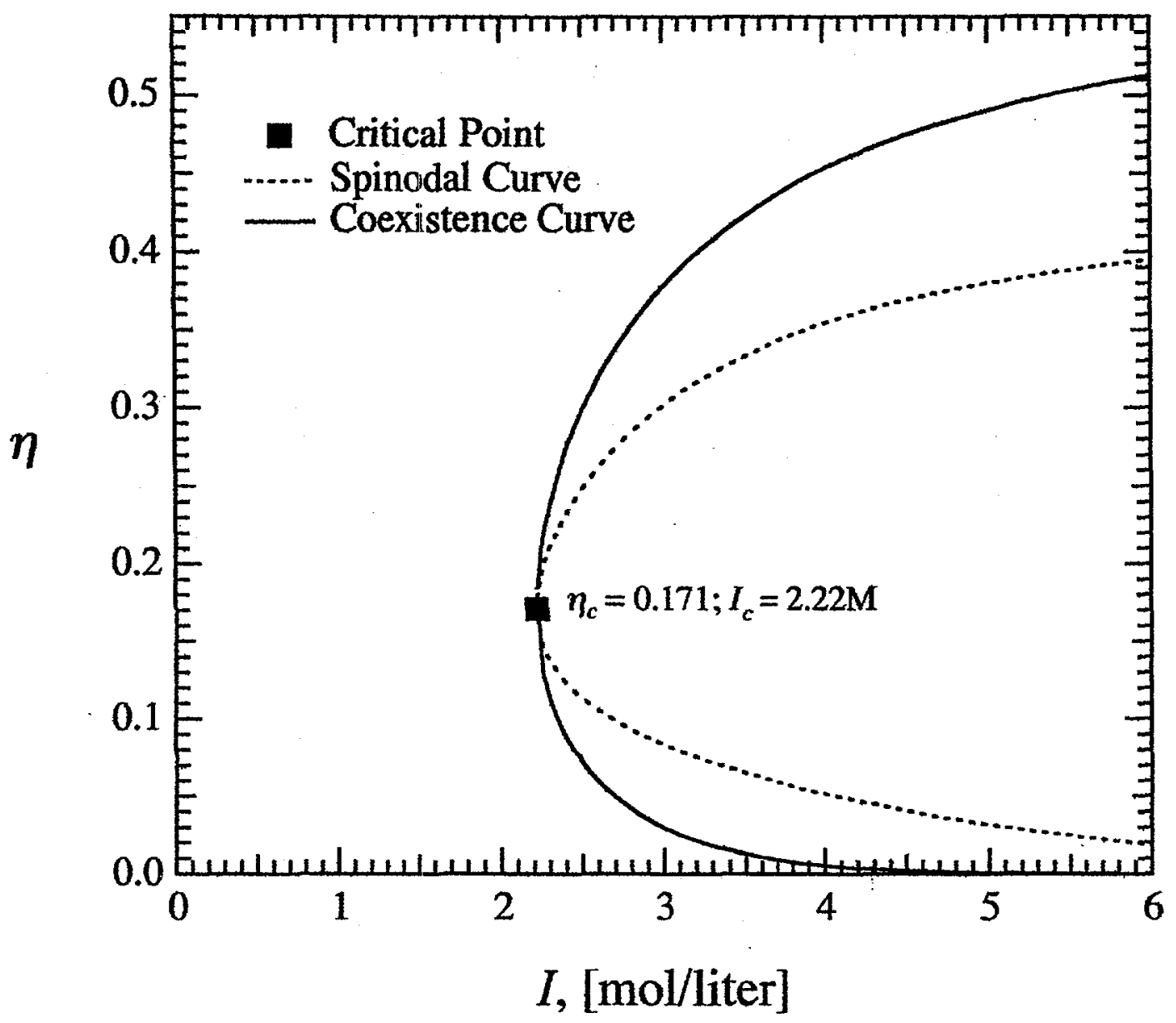

Figure 7: Typical critical point and spinodal and coexistence curves calculated from the RPA-SAFT equation of state.

Protein parameters: $\quad \sigma_{p}=50 \AA, H / k_{B} T=10, M=6, \varepsilon_{s p} / k_{B} T=2, V=0.02$

Salt parameters: $\quad \sigma_{c a t}=6.62 \AA, z_{c a t}=1$

$\sigma_{a n}=7.58 \AA, z_{a n}=-2$ 

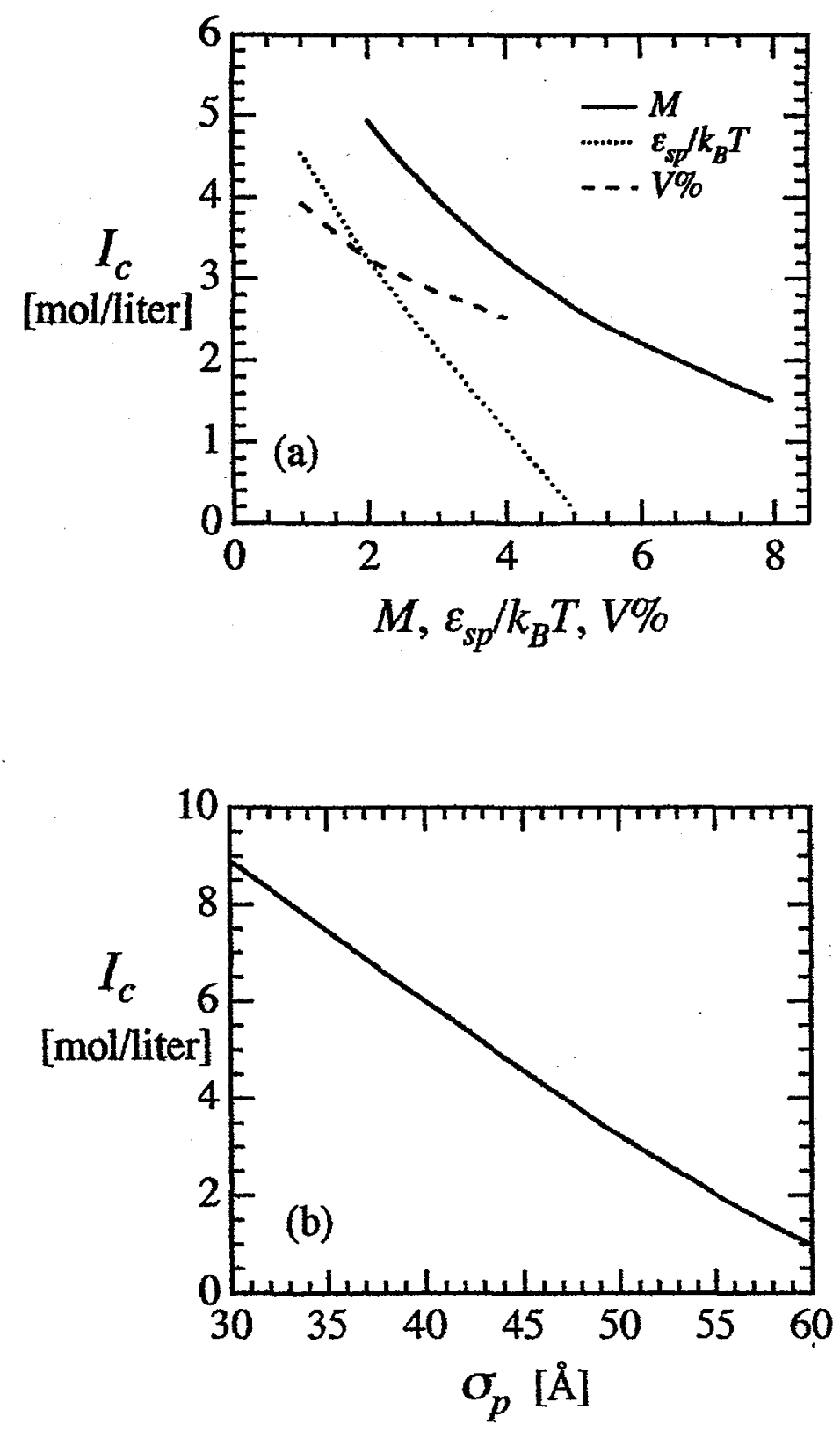

Figure 8: Dependence of critical ionic strength, $I_{c}$, on:

(a) SAFT parameters $M, \varepsilon_{s p} / k_{B} T$, and $V \%=V \cdot 100$

(b) protein diameter $\sigma_{p}$ 

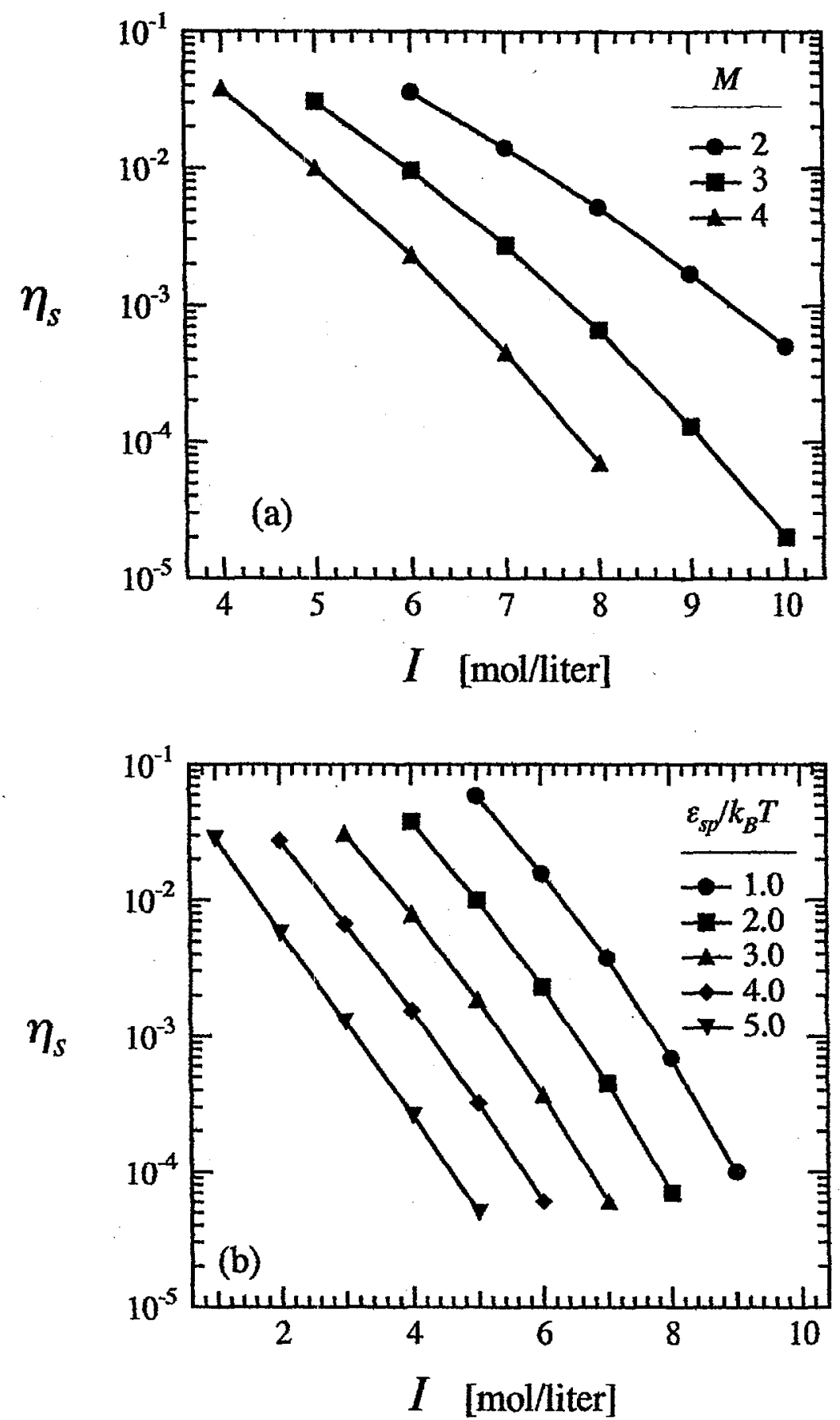

Figure 9: Dependence of equilibrium protein packing fraction in the supernatant phase, $\eta_{s}$, on SAFT parameters: (a) $M$, and (b) $\varepsilon_{s p} / k_{B} T$ in aqueous ammonium sulfate solutions.

Protein parameters: $\sigma_{p}=50 \AA, H / k_{B} T=10, M=4$ [in (b)], $\varepsilon_{s p} / k_{B} T=2$ [in (a)], $V=0.02$ Salt parameters:

$$
\begin{aligned}
& \sigma_{c a t}=6.62 \AA, z_{c a t}=1 \\
& \sigma_{a n}=7.58 \AA, z_{a n}=-2
\end{aligned}
$$



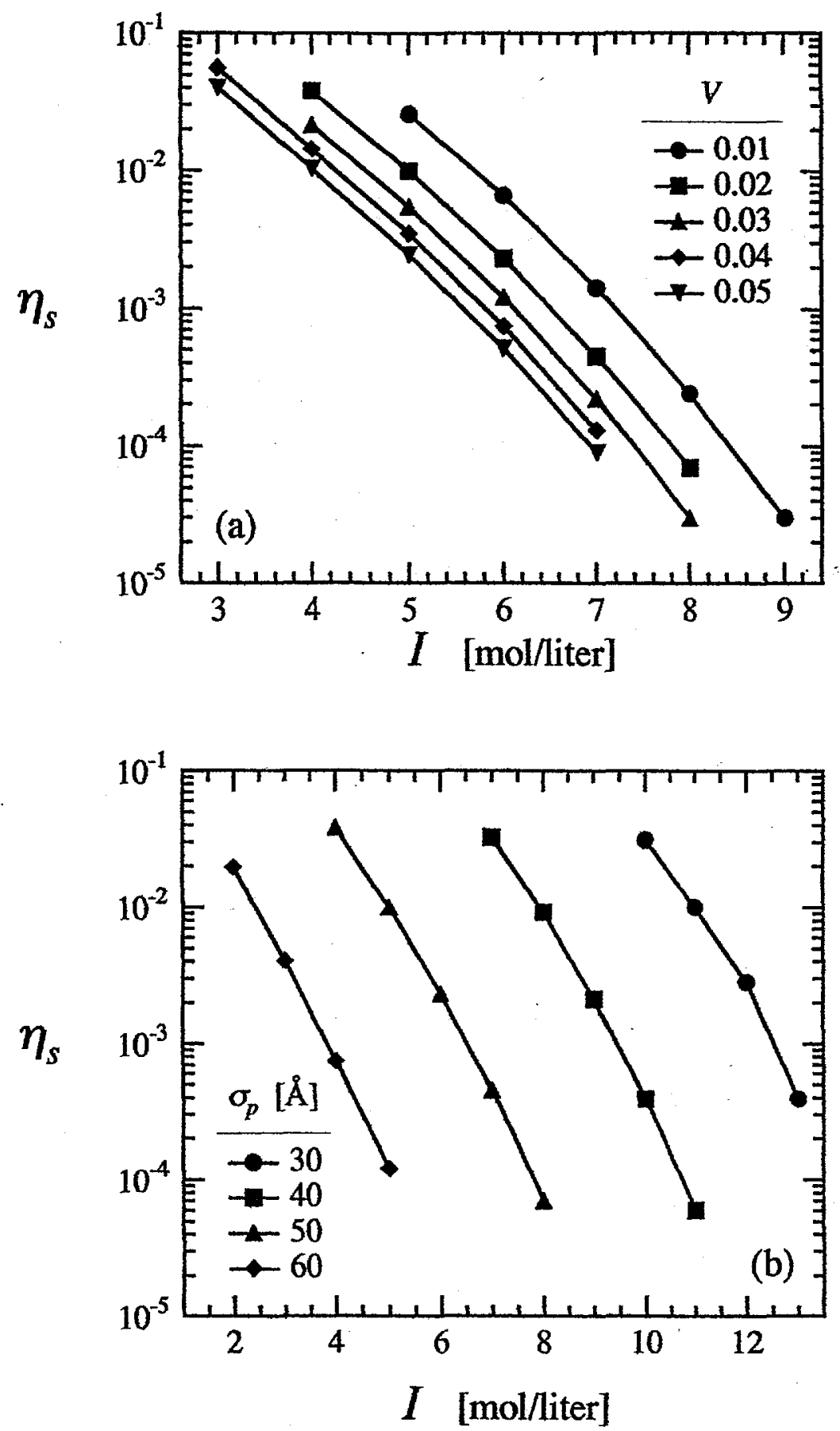

Figure 10: Dependence of equilibrium protein packing fraction in the supernatant phase, $\eta_{s}$ on: (a) SAFT parameter $V$, and (b) $\sigma_{p}$ in aqueous ammonium sulfate solutions.

Protein parameters: $\quad \sigma_{p}=50 \AA$ [in (a)], $H / k_{B} T=10, M=4, \varepsilon_{s p} / k_{B} T=2, V=0.02$ [in (b)] Salt parameters:

$$
\begin{aligned}
& \left.\sigma_{p}=6.1 \mathrm{i}(\mathrm{a})\right], \\
& \sigma_{c a t}=6.62 \AA, z_{c a t}=1 \\
& \sigma_{a n}=7.58 \AA, z_{a n}=-2
\end{aligned}
$$



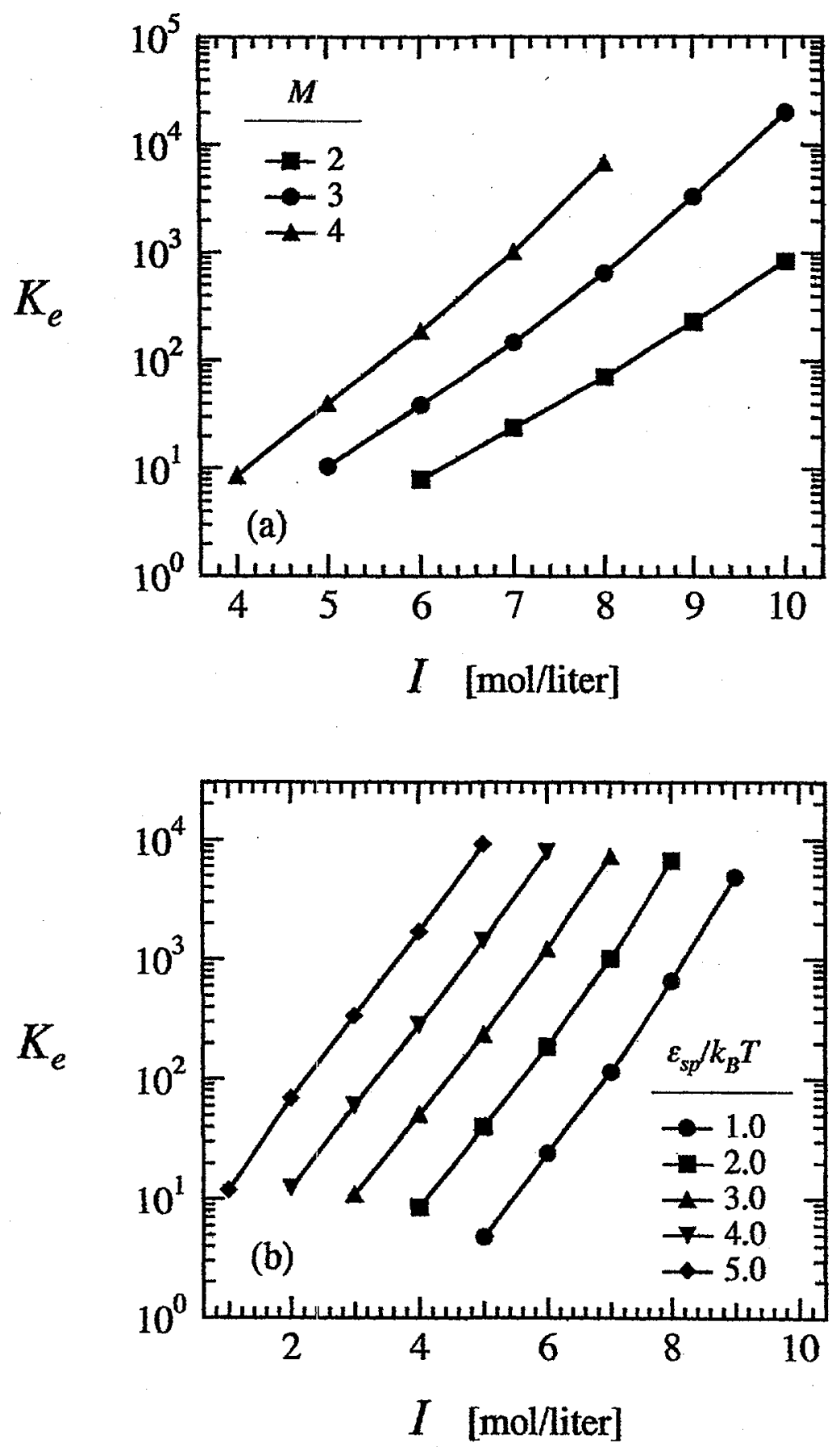

Figure 11: Dependence of protein equilibrium partition coefficient, $K_{e}$, on SAFT parameters: (a) $M$, and (b) $\varepsilon_{s p} / k_{B} T$ in aqueous ammonium sulfate solutions.

Protein parameters: $\quad \sigma_{p}=50 \AA, H / k_{B} T=10, M=4\left[\right.$ in (b)], $\varepsilon_{s p} / k_{B} T=2$ [in (a)], $V=0.02$ Salt parameters:

$$
\begin{aligned}
& \sigma_{c a t}=6.62 \AA, z_{c a t}=1 \\
& \sigma_{a n}=7.58 \AA, z_{a n}=-2
\end{aligned}
$$



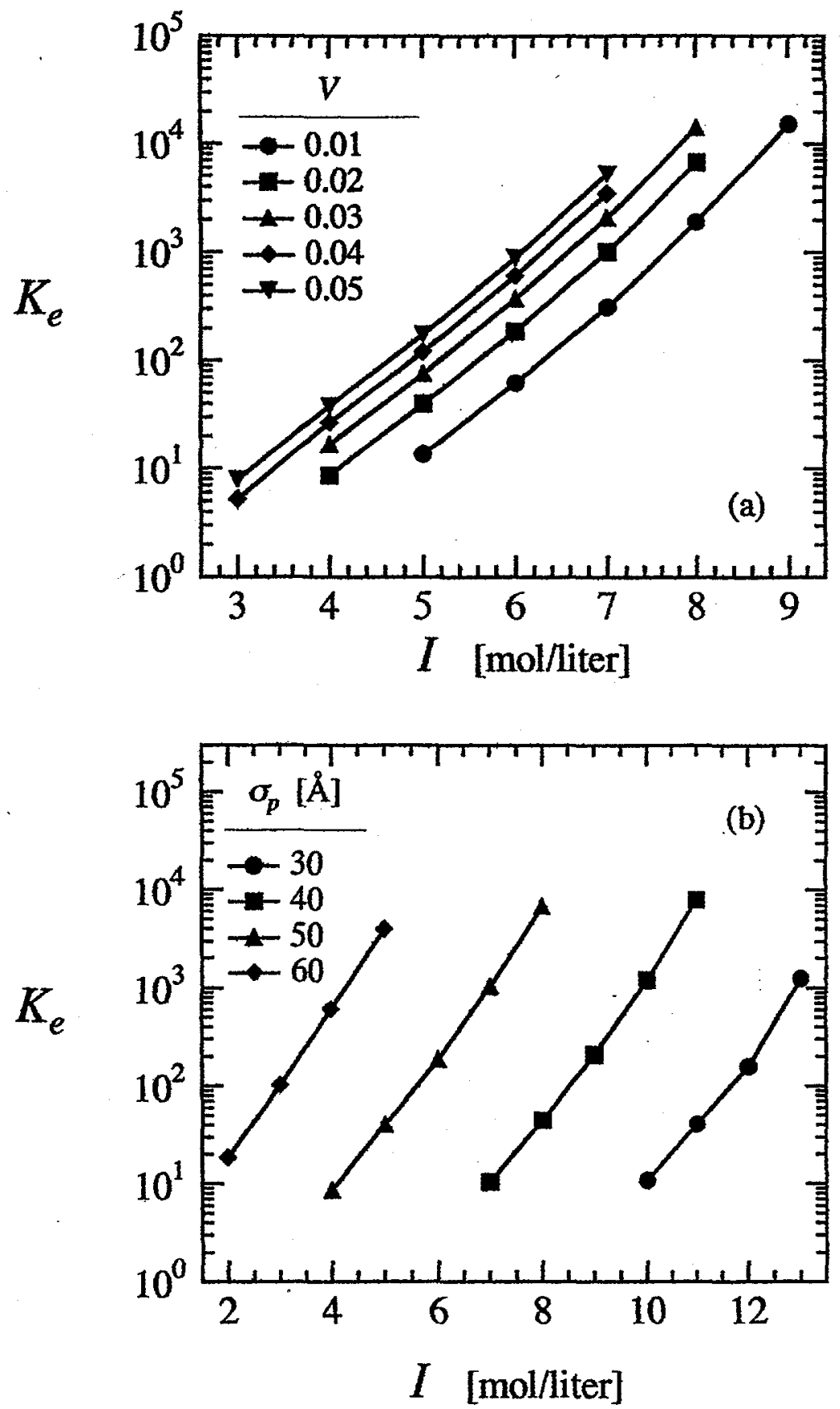

Figure 12: Dependence of protein equilibrium partition coefficient, $K_{e}$, on:

(a) SAFT parameter $V$, and

(b) $\sigma_{p}$ in aqueous ammonium sulfate solutions.

Protein parameters: $\quad \sigma_{p}=50 \AA$ [in (a)], $H / k_{B} T=10, M=4, \varepsilon_{s p} / k_{B} T=2, V=0.02$ [in (b)] Salt parameters: $\quad \sigma_{c a t}=6.62 \AA, z_{c a t}=1$$$
\sigma_{a n}=7.58 \AA, z_{a n}=-2
$$ 


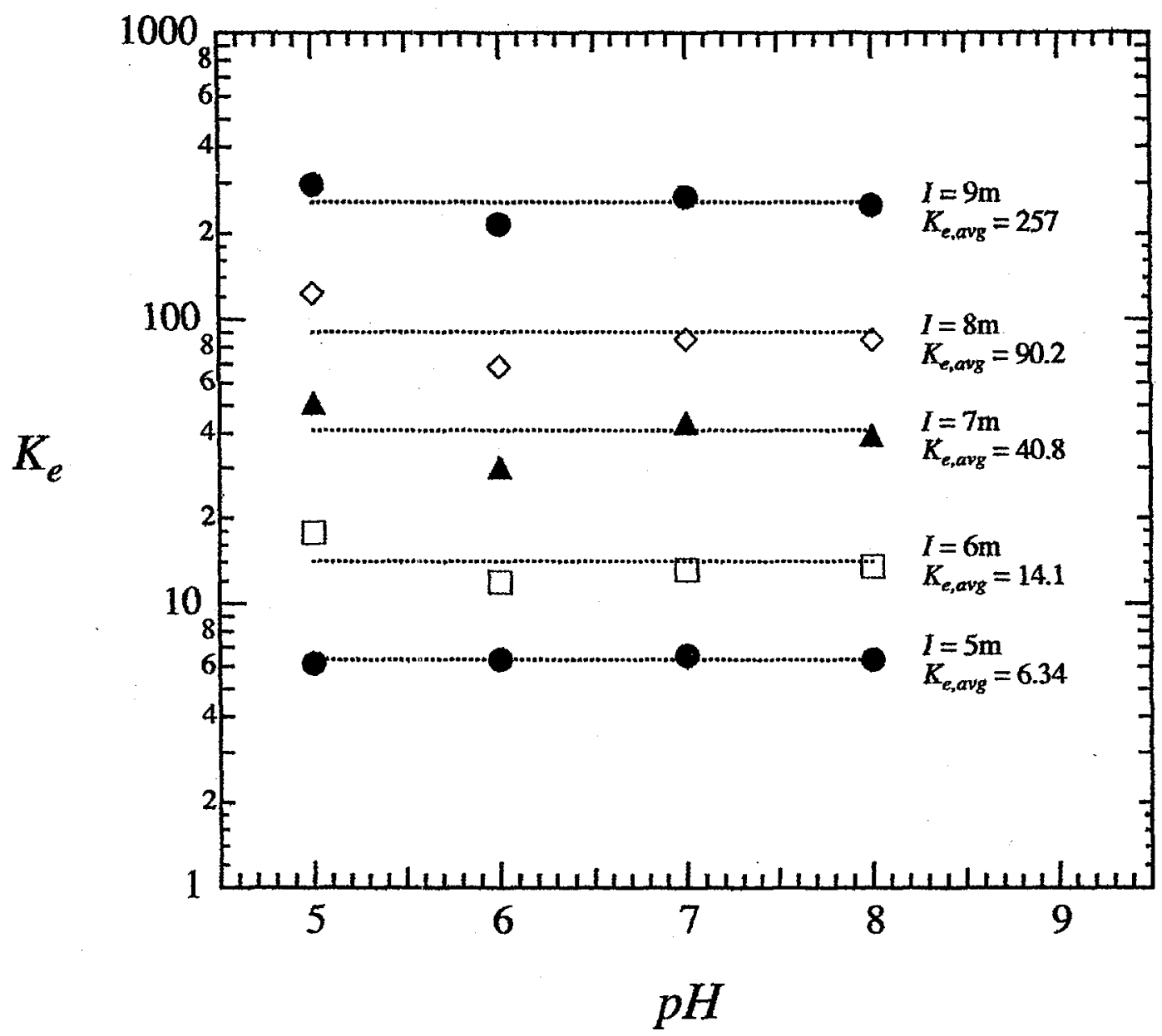

Figure 13: Dependence of the equilibrium protein partition coefficient, $K_{e}$, on $\mathrm{pH}$ and ionic strength for lysozyme precipitating in solutions of ammonium sulfate at $298 \mathrm{~K}$. 


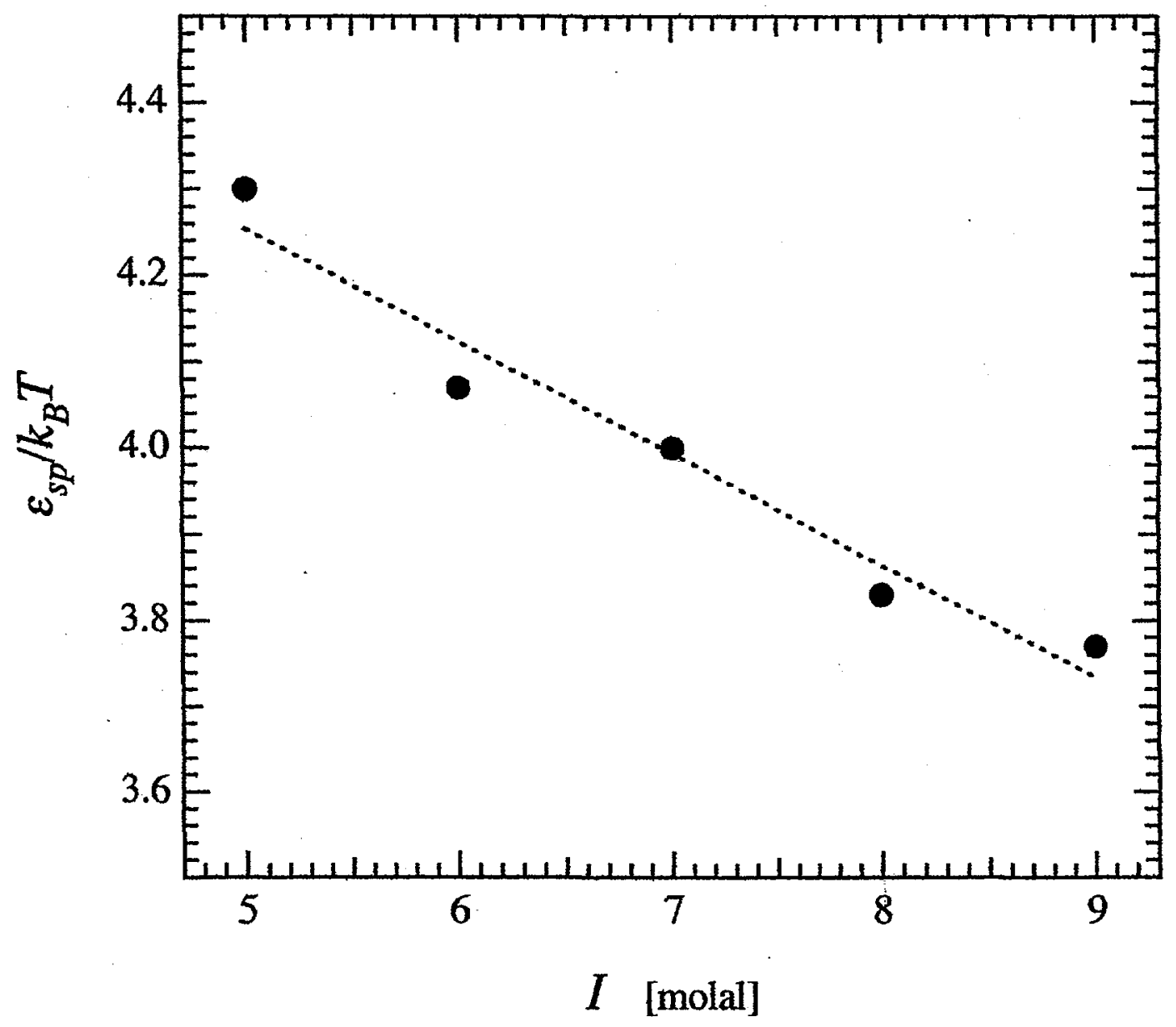

Figure 14: Dependence of specific-interaction energy, $\varepsilon_{s p} / k_{B} T$, on solution ionic strength for precipitation of hen-egg-white lysozyme in solutions of ammonium sulfate at $298 \mathrm{~K}$.

Ammonium sulfate: $\quad \sigma_{c a t}=6.62 \AA, \sigma_{a n}=7.58 \AA ; z_{c a t}=+1, z_{a n}=-2$ Lysozyme: $\quad \sigma_{p}=34.4 \AA, H / k_{B} T=8.9, M=6, V=0.03$ 

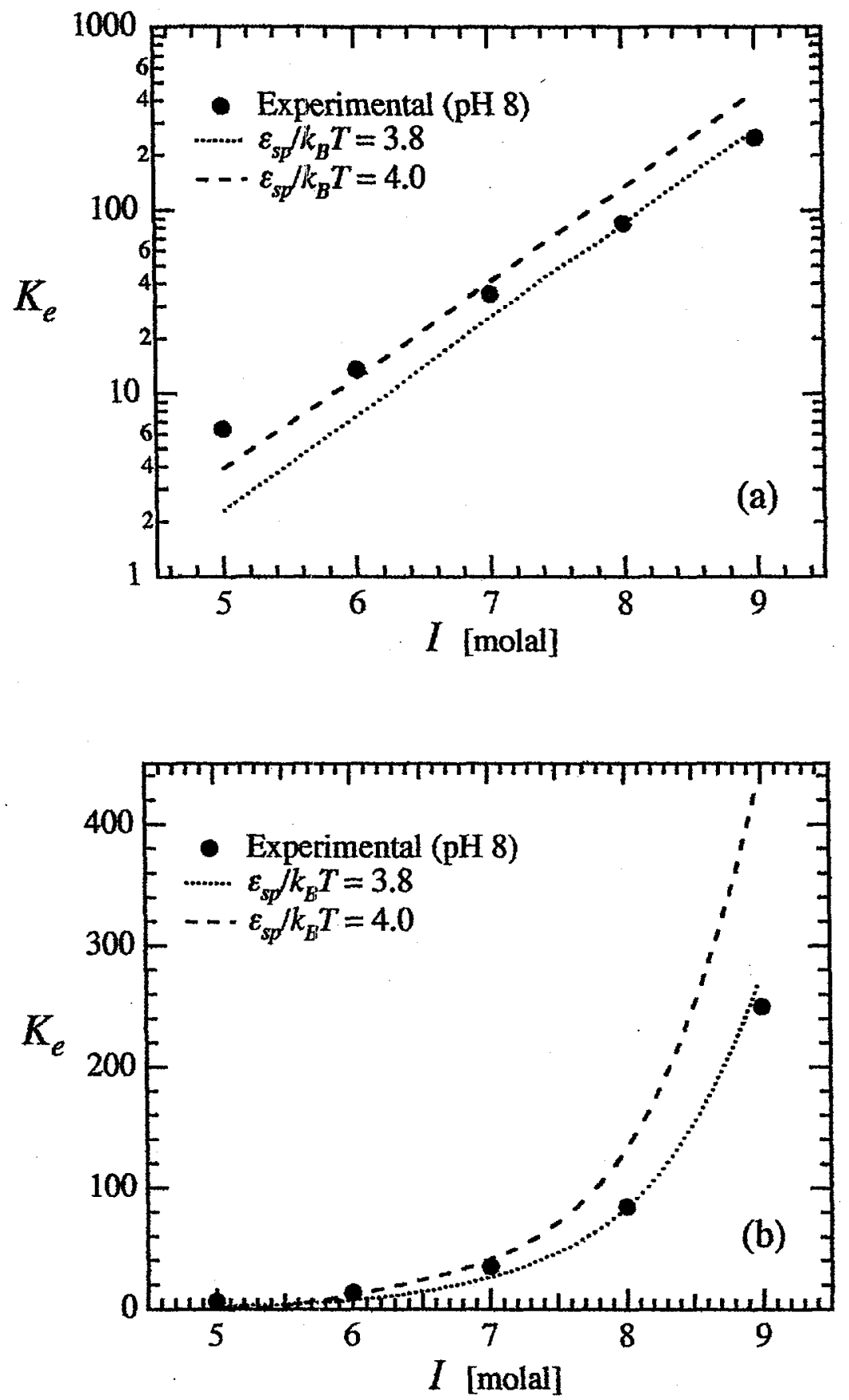

Figure 15: Experimental and calculated values of the equilibrium protein partition coefficient, $K_{e}$, as a function of ionic strength for hen-egg-white lysozyme precipitating in solutions of ammonium sulfate at $298 \mathrm{~K}$, in logarithmic (a) and linear (b) scale.

Ammonium sulfate: $\quad \sigma_{c a t}=6.62 \AA, \sigma_{a n}=7.58 \AA ; z_{c a t}=+1, z_{a n}=-2$

Lysozyme:

$$
\begin{aligned}
& \sigma_{c a t}=6.62 \AA, \sigma_{a n}=7.58 \AA ; z_{c a t}=+1, z_{a n}=-2 \\
& \sigma_{p}=34.4 \AA, H / k_{B} T=8.9, M=6, \varepsilon_{s p} / k_{B} T=3.8, V=0.03
\end{aligned}
$$



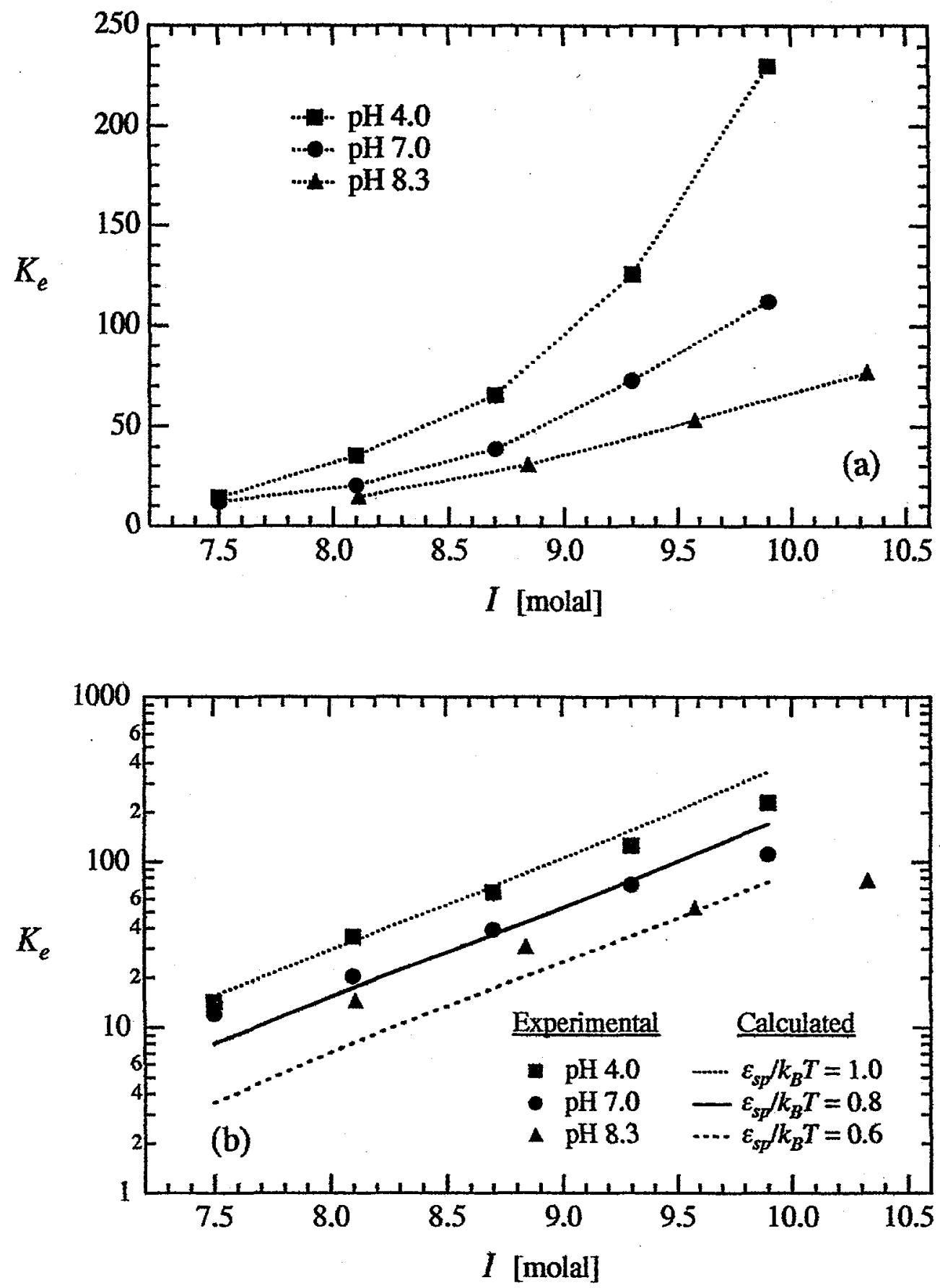

Figure 16: Dependence of the protein equilibrium partition coefficient, $K_{e}$, on ionic strength and $\mathrm{pH}$ for $\alpha$-chymotrypsin in solutions of ammonium sulfate at $298 \mathrm{~K}$.

(a) Experimental data displayed in linear format

(b) experimental and calculated values of $K_{e}$ displayed in logarithmic format.

Ammonium sulfate: $\quad \sigma_{c a t}=6.62 \AA, \sigma_{a n}=7.58 \AA ; z_{c a t}=+1, z_{a n}=-2$ $\alpha$-chymotrypsin: $\quad \sigma_{p}=43.4 \AA, H / k_{B} T=10, M=6, V=0.02$ 


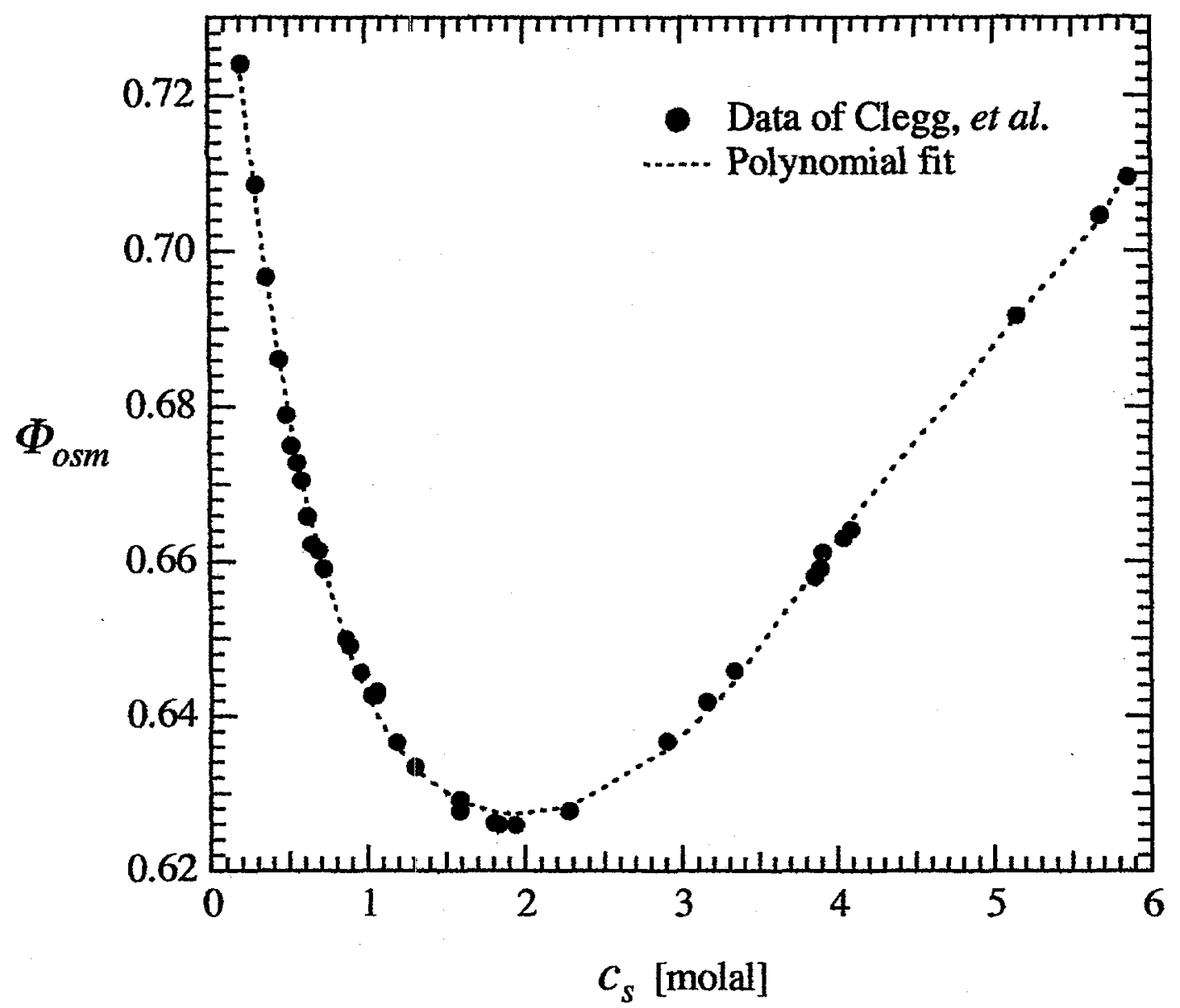

Figure B.1: Osmotic coefficients in pure aqueous solutions of ammonium sulfate at $298.15 \mathrm{~K}$. 


\section{LITERATURE CITED}

Albertsson, P.-A. (1986). Partition of Cell Particles and Macromolecules. New York, Wiley.

Arakawa, T. and S. N. Timasheff, "Preferential interactions of proteins with salts in concentrated solutions," Biochem. (1982) 21: 6545-6552.

Arakawa, T. and S. N. Timasheff, "Mechanism of protein salting-in and salting-out by divalent cation salts: Balance between hydration and salt binding," Biochem. (1984) 23: 5912.

Arakawa, T. and S. N. Timasheff, "Theory of protein solubility," Methods Enzymol. (1985) 114: 49-.

Asakura, S. and F. Oosawa, "On interaction between two bodies immersed in a solution of macromolecules," J. Chem. Phys. (1954) 22: 1255-1256.

Asakura, S. and F. Oosawa, "Interaction between particles suspended in solutions of macromolecules," J. Poly. Sci. (1958) 33: 183.

Carnahan, N. and K. Starling, "Thermodynamic properties of a rigid-sphere fluid," Chem. Phys. (1970) 53: 600.

Carr, C., "Studies on the binding of small ions in protein solutions with the use of membrane electrodes. III. The binding of chloride ions in solutions of various proteins," Arch. Biochem. Biophys. (1953) 46: 417-423.

Carr, C., "Studies on the binding of small ions in protein solutions with the use of membrane electrodes. IV. The binding of calcium ions in solutions of various proteins," Arch. Biochem. Biophys. (1953) 40: 423-431.

Chiew, Y. C., D. E. Kuehner, H. W. Blanch and J. M. Prausnitz, "Molecular thermodynamics of salt-induced protein precipitation," AIChE J. (1995) 41(9): 21502159. 
Clegg, S. L., S. Milioto and D. A. Palmer, "Osmotic and activity coefficients of aqueous $\left(\mathrm{NH}_{4}\right)_{2} \mathrm{SO}_{4}$ as a function of temperature, and aqueous $\left(\mathrm{NH}_{4}\right)_{2} \mathrm{SO}_{4}-\mathrm{H}_{2} \mathrm{SO}_{4}$ mixtures at 298.15 K and 323.15 K," J. Chem. Eng. Data (1996) 41: 455-467.

Coen, C. J., "Molecular Thermodynamics of Protein Interactions and Phase Behavior in Aqueous Electrolyte Solutions," Ph.D. thesis. Department of Chemical Engineering, University of California, Berkeley (1995).

Curtis, R. A., J. M. Prausnitz and H. W. Blanch, "Protein-protein and protein-salt interactions in aqueous protein solutions containing concentrated electrolyte," Biotech. Bioeng. (1998) 57(1): 11-21.

Edsall, J. T. (1943). Proteins as Acids and Bases. Proteins, Amino Acids, and Peptides. E. J. Cohn and J. T. Edsall. New York, Reinhold.

Fergg, F., "Hydrogen-Ion Titrations of Amino Acids and Proteins in Solutions Containing Concentrated Electrolyte," Dipl. Ing. thesis. Department of Chemical Engineering, University of California, Berkeley (1994).

Foster, P. R., P. Dunhill and M. D. Lilly, "The precipitation of enzymes from cell extracts of Saccharomyces Cerevisiae by polyethylene glycol," Biochem. Biophys. Acta (1973) 317: 505.

Green, A. A., "Studies of the physical chemistry of the proteins, VIII: The solubility of hemoglobin in concentrated salt solutions," I. Biol. Chem. (1931) 93: 495.

Green, A. A., "Studies of the physical chemistry of the proteins, X: The solubility of hemoglobin in solutions of chlorides and sulfates of varying concentration," J. Biol. Chem. (1932) 95: 47.

Grimson, M. J., "Small angle scattering from colloidal dispersions," J. Chem. Soc. Farad. Trans. II (1983) 79: 817.

Haire, R. N., W. A. Tisel, J. G. White and A. Rosenberg, "On the precipitation of proteins by polymers: The hernoglobin polyethylene glycol system," Biopol. (1984) 23: 2761. 
Hamaker, H. C., "The London-van-der Waals attraction between spherical particles," Physica A (1937) 4(10): 1058-1072.

Hill, T. L. (1960). An Introduction to Statistical Thermodynamics. New York, Dover Publications.

Hofmeister, F., "Zur lehre von der wirkung der salze," Arch. Expt. Pathol. Pharmakol. (1888) 24: 247-260.

Horvath, A. L. (1985). Handbook of Aqueous Electrolyte Solutions: Physical Properties, Estimation and Correlation Methods. Chichester, Ellis Horwood.

Israelachvili, J. N. (1992). Intermolecular and Surface Forces. London, Academic Press.

Israelachvili, J. N. and G. E. Adams, J. Chem. Soc. Farad. Trans. I (1978) 74: 975.

Jackson, G., W. G. Chapman and K. E. Gubbins, "Phase equilibria of associating fluids: Spherical molecules with multiple bonding sites," Molec. Phys. (1988) 64: 1.

Konior, J. and C. Jedrzejek, "Analytic formulations of the WCA-type perturbation theory for a hard-core-with-one-Y ukawa-tail fluid," Molec. Phys. (1985) 55: 187.

Kuehner, D., J. Engmann, F. Fergg, M. Wernick, H. W. Blanch and J. M. Prausnitz, "Lysozyme net charge and ion binding in aqueous electrolyte solutions," in preparation (1998) .

Kuehner, D. E., H. W. Blanch and J. M. Prausnitz, "Salt-induced protein precipitation: phase equilibria from an equation of state," Fluid Phase Equil. (1996) 11 6: 140-147.

Kuehner, D. E., C. Heyer, C. Rämsch, U. M. Fornefeld, H. W. Blanch and J. M. Prausnitz, "Interactions of lysozyme in concentrated electrolyte solutions from dynamic light-scattering measurements," Biophys. J. (1997) 73: 3211-3224.

Mahadevan, H. and C. K. Hall, "Statistical-mechanical model of protein precipitation by nonionic polymer," AIChE J. (1990) 36: 1517. 
Mahadevan, H. and C. K. Hall, "Theory of precipitation of protein mixtures by nonionic polymer," AIChE J. (1992) 38(4): 573-591.

McGregor, W. C., "Large scale isolation and purification of proteins from recombinant $E$. Coli," Ann. N. Y. Acad. Sci. (1983) 413: 231 -.

McQuarrie, D. (1976). Statistical Mechanics. New York, Harper-Collins.

Melander, W. and C. Horvath, "Salt effects on hydrophobic interactions in precipitation and chromatography of proteins: An interpretation of lyotropic series," Arch. Biochem. Biophys. (1977) 183: 200-215.

Nir, S., "Van der Waals interactions between surfaces of biological interest," Prog. Surf. Sci. (1976) 8: 1.

Ornstein, L. S. and F. Zernike, Prok. Acad. Sci. (1914) 17: 793.

Pitzer, K. S. (1991). Activity Coefficients in Electrolyte Solutions. Boca Raton, CRC Press.

Prausnitz, J. M., R. N. Lichtenthaler and E. G. Azevedo (1986). Molecular Thermodynamics of Fluid-Phase Equilibria. Engleweood Cliffs, NJ, Prentice-Hall.

Przybycien, T. M. and J. Bailey, "Solubility-activity relationship in the inorganic saltinduced precipitation of $\alpha$-chymotrypsin," Enzyme Microb. Tech. (1989) 11: 264.

Ries-Kautt, M. and A. Ducruix, "Crystallization of previously desalted lysozyme in the presence of sulfate ions," Acta Cryst. (1994) D50: 366-369.

Ries-Kautt, M. A. and A. F. Ducruix, "Relative effectiveness of various ions on the solubility and crystal growth of lysozyme," J. Biol. Chem. (1989) 264: 745-748.

Rosenbaum, D., P. C. Zamora and C. F. Zukoski, "Phase behavior of small attractive colloidal particles," Phys, Rev. Lett. (1996) 76(1): 150-153. 
Rothstein, F. (1994). Differential Precipitation of Proteins. Protein Purification Process Engineering. R. G. Harrison. New York, Dekkér.

Rothstein, F. (1994). Protein Purification Process Engineering. New York, Dekker.

Shih, Y.-C., H. W. Blanch and J. M. Prausnitz, "Some characteristics of protein precipitation by salts," Biotech. Bioeng. (1992) 40: 1155-1164.

Stryer, L. (1988). Biochemistry. New York, W.H. Freeman.

Tavares, F. W. and S. I. Sandler, "Phase equilibrium for the mean-force potential of globular protein solutions," AIChE J. (1997) 43(1): 218-231.

Verwey, E. J. W. and J. T. G. Overbeek (1948). Theory of Stability of Lyophobic Colloids. Amsterdam, Elsevier.

Vlachy, V., H. W. Blanch and J. M. Prausnitz, "Liquid-liquid phase separations in aqueous solutions of globular proteins," AIChE J. (1993) 39(2): 215-223.

Wertheim, M. S., "Fluids with highly directional attractive forces. III. multiple attraction sites," J. Stat. Phys. (1986a) 42: 459.

Wertheim, M. S., "Fluids with highly directional attractive forces. IV. equilibrium polymerization," J. Stat. Phys. (1986b) 42: 477. 